\title{
Chiral gravitational effect in time-dependent backgrounds
}

\author{
Kohei Kamada, ${ }^{a}$ Jun'ya Kume ${ }^{a, b}$ and Yusuke Yamada ${ }^{a}$ \\ ${ }^{a}$ Research Center for the Early Universe (RESCEU), Graduate School of Science, \\ The University of Tokyo, \\ Hongo 7-3-1 Bunkyo-ku, Tokyo 113-0033, Japan \\ ${ }^{b}$ Department of Physics, Graduate School of Science, The University of Tokyo, \\ Hongo 7-3-1 Bunkyo-ku, Tokyo 113-0033, Japan \\ E-mail: kohei.kamada@resceu.s.u-tokyo.ac.jp, \\ kjun0107@resceu.s.u-tokyo.ac.jp, yamada@resceu.s.u-tokyo.ac.jp
}

ABSTRACT: Gravitational counterpart of the chiral magnetic effect, which is referred as the chiral gravitational effect, can also be of interest in a cosmological setup. In this study, we investigate this effect in the time-dependent chiral asymmetric fermion background and in the expanding spacetime by formulating the effective action of gravitational waves. We also analyze the anomaly equation to see how the backreaction from gravitational waves to thermal chiral plasma occurs. We find that the non-trivial time dependence of chiral chemical potential, which can be induced in some scenarios of baryogenesis, is the key ingredient of the chiral gravitational effect. It turns out that the "memory" of the effect is imprinted on the high frequency gravitational waves propagating in the plasma. Cosmological implications and potential effects on the gravitational wave observation are briefly discussed.

Keywords: Anomalies in Field and String Theories, Classical Theories of Gravity, Cosmology of Theories beyond the SM

ArXIV EPRINT: 2104.00583 


\section{Contents}

1 Introduction 1

2 Chiral gravitational effect in a dynamical background 4

3 Gravitational birefringence and the backreaction to chiral plasma in the Minkowski background

3.1 Propagation of gravitational waves

$\begin{array}{lll}3.2 & \text { Backreaction to chiral plasma from gravitational waves } & 10\end{array}$

4 Chiral gravitational effect in the expanding background $\quad 16$

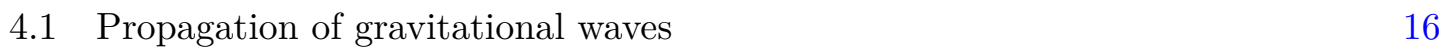

$\begin{array}{ll}\text { 4.2 The effect of the diluting chemical potential } & 18\end{array}$

5 Discussion $\quad 21$

\section{Introduction}

Parity violating effects related to quantum anomalies have been discussed in various fields of physics, from condensed matter to cosmology. One of the representative examples is the "Chiral Magnetic Effect"(CME) [1-5], which arises in the theory with a U(1) gauge symmetry. The CME is a phenomenon where the magnetic field induces the electric current parallel to the field itself, under the presence of the asymmetry between left- and right-handed fermions or the chiral asymmetry, which give rise to the distinct transport phenomena. Since the chiral anomaly is ubiquitous in quantum field theory, it is expected to appear in a broad range of energy scales, such as in the Weyl semimetals [6-12], relativistic heavy ion collisions [13-20], or compact stars [21-27]. Moreover, in the hot early Universe, where strong magnetic fields [28] as well as chiral asymmetry [29] can exist, interesting phenomena can take place. Since the current induced by the CME leads to the instability in the magnetic fields in chiral plasma, known as the chiral plasma instability (CPI) [30, 31], it is, e.g., ascribed as the possible origin of the primordial magnetic fields [30, 32], which leads to the baryogenesis [33] from the hypermagnetic helicity decay [34-39]. Numerical magnetohydrodynamic simulations $[27,40]$ as well as lattice simulations $[41,42]$ have recently been performed to show how the CPI proceeds in thermal environments. Since the amplified electromagnetic fields contribute to the transverse and traceless part of the energy momentum tensor, gravitational waves are emitted [43, 44], which is one of the interesting consequences of the effect. The primordial chiral asymmetry, the key ingredient in these processes, can be generated in well-motivated cosmological phenomena such as the grand unified theory (GUT) baryogenesis and axion inflation [33, 45-47]. 
While the cosmological application of the CME is now being investigated in detail, its gravitational counterpart referred as "chiral gravitational effect" [48] has not been extensively investigated so far. In the case of chiral gravitational effect, the graviton (the transverse and traceless part of the metric perturbation) receives parity-violating vacuum polarization from the chiral asymmetry in the system, which leads to the anomalous contribution to the energy momentum tensor. ${ }^{1}$ Note that parity-violating effect is ascribed to the "gravitational (chiral) anomaly" $[50]^{2}$ in this case. Applications and discussions of this anomaly in cosmology have mainly focused on the study of leptogenesis, in which it connects the lepton chiral charge to the helicity of gravitational waves. This is realized in pseudo-scalar inflation models with the gravitational Chern-Simons term, where the chiral gravitational waves and the primordial lepton asymmetry are simultaneously generated during inflation through this anomaly $[52-55] .^{3}$ In this simple setup, unfortunately, the resultant lepton asymmetry is too small to explain matter anti-matter asymmetry of the Universe [54, 57], though. ${ }^{4}$ On the other hand, the chiral gravitational effect should also be able to cause interesting phenomena in the primordial thermal plasma with net chiral asymmetry, in a similar way to the CPI. Namely, the anomalous energy momentum tensor induced by the chiral gravitational effect $[64,65]$ modifies the propagation of gravitational waves, and it in turn affects the fermion background through the anomaly. Thus, one can expect non-trivial interplay between the chiral imbalance of the plasma and the gravitational wave in the early Universe.

Before investigating the chiral gravitational effect in realistic cosmological setups, one needs to extend the formulation in the previous study. One of the pioneer works on the chiral gravitational effect [48] considered this effect in the static thermal fermion background with a chiral asymmetry, based on refs. $[64,65]$ where an anomalous dispersion relation of the gravitational waves in such a background is derived. To investigate this effect in the primordial chiral thermal plasma, one needs to go beyond the static background since the chiral asymmetry characterized by the chemical potential $\mu_{5}$ exhibits a non-trivial time dependence at the generation and decay. The former typically takes place at some baryogenesis scenarios such as the GUT baryogenesis [33, 47], whereas the latter happens when the electron Yukawa interaction gets in equilibrium [29]. Note that unlike vector fields, gravitons are not conformally invariant, and hence the dilution due to the cosmic expansion also affects the phenomena induced by the chiral gravitational effect. In this study, we provide the effective field theoretic view point to incorporate the time dependence of the fermion background with a chiral imbalance and that of the background geometry into the chiral gravitational effect.

A theoretical aspect of the CME is that the effect of the chiral anomaly on the gauge field dynamics can be understood as the induced Chern-Simons term in the effective La-

\footnotetext{
${ }^{1}$ See also ref. [49] for the study on the chiral gravitational effect from the holographic point of view.

${ }^{2}$ The chiral vortical effect partially originates from the gravitational chiral anomaly on the vector part of the metric perturbation [51].

${ }^{3}$ See also ref. [56] for the case where the Gauss-Bonnet term contribution is incorporated in this model.

${ }^{4}$ Gravitaional leptogenesis can be successful in the models where the inflaton couples to the ChernPontryagin density of the gauge fields [58-62]. See also ref. [63] where the effect of lepton number violation due to the Majorana neutrino mass has been discussed.
} 
grangian, which can be obtained by integrating out the fermions coupled to finite chiral chemical potential [66]. In this effective Lagrangian, an asymmetric state of thermal plasma can be expressed by introducing a spurious scalar field $\theta(x)$ which anomalously couples to the Chern-Pontryagin density $[67,68]$. This formulation of the effective field theory studied in the CME can enable us to incorporate the time-dependent behavior of the background and we here apply the similar procedure to the chiral gravitational effect. Note that a similar discussion has been studied in ref. [69] where the authors consider the effect of an asymmetric fermion background, namely the cosmic neutrino background, to the propagating gravitational wave during the matter-dominated era, through the gravitational Chern-Simons term in the effective Lagrangian. Nevertheless, we should note that there are non-local thermal and chemical potential corrections found in refs. [64, 65], which cannot be described within the local Lagrangian approach. We will focus on relatively high momentum gravitational waves, for which non-local effects are negligible or subdominant.

To investigate comprehensively the evolution of the chiral plasma and gravitational waves under the presence of the gravitational anomaly, we need to take into account the backreaction on the chiral plasma, which is another subject of this study. As a first step, we analyze simple toy models in the following way: first, we derive the gravitational wave solutions perturbatively corrected by the gravitational Chern-Simons term both in Minkowski spacetime and Friedmann-Robertson-Walker (FRW) background, which clarify how the chiral gravitational effect affects the propagation of gravitational waves. We here assume that background gravitational waves have already existed in the system, which is motivated by the stochastic gravitational wave background from such as inflation [70, 71], preheating [72-75], and first-order phase transitions [76-79]. Then we consider its backreaction to the chiral plasma through the anomaly equation for models in both Minkowski and FRW backgrounds. Note that we restrict ourselves into the region where the chiral chemical potential and the effect of the Chern-Simons term is sufficiently small, to avoid the ghost instability, which will be explained later.

One of the main results of our investigation, explicitly shown in the Minkowski spacetime analysis, is that the propagation of gravitational wave is affected only when the chiral chemical potential $\mu_{5}$ has non-trivial time dependence. Such a behavior of the chiral gravitational effect is in contrast with the case of CME where the instability is triggered simply by the presence of non-vanishing constant $\mu_{5} .{ }^{5}$ We emphasize that the expansion of the Universe non-trivially contributes to the chiral gravitational effect since gravitons are not conformal. As we will see, the expansion leads to effectively time dependent chemical potential, which triggers non-trivial interplay between the gravitational waves and the chiral chemical potential through the chiral gravitational effect. We also demonstrate that the time dependence of chemical potential generically leaves the imprints of the primordial chirality violation on gravitational waves. Then we show that the backreaction from gravitational waves to chiral plasma is typically small compared to the dominant part in chemical potential dynamics. However, when the dominant part eventually decays, the "imprints" at

\footnotetext{
${ }^{5}$ When one considers a time-dependent pumping of electrons in asymmetric Weyl semimetals, the anomalous transport is characterized by the time derivative of $\mu_{5}$ even in the case of CME [80].
} 
an earlier time can become important for late time behavior of chemical potential. There, the backreaction leads to the oscillatory conversion between the chiral chemical potential and the helicity of gravitational waves driven by the external chirality-changing process.

The rest of the paper is organized as follows. In section 2, we make a brief review of the derivation of the gravitational Chern-Simons term in the effective Lagrangian arising from chiral asymmetric fermion background. Then we discuss its general physical property that affects the propagation of the gravitational waves as a consequence of chiral gravitational effect. By incorporating the induced Chern-Simons term into the equation of motion, we explicitly show the corrected gravitational waves in time-dependent chiral plasma for the Minkowski spacetime and evaluate its backreaction through the anomaly equation in section 3. In section 4, we consider the FRW spacetime to investigate the effect of cosmological expansion on the chiral gravitational effect. In the same way as in the Minkowski spacetime case, we solve the modified equation of motion and then evaluate the backreaction from gravitational waves to the chiral plasma through the gravitational anomaly. The final section is devoted to the summary and discussion. Throughout this paper, we will use the convention in ref. [81], e.g., $\eta_{\mu \nu}=\operatorname{diag}(-1,1,1,1)$ and the Levi-Civita symbol $\epsilon^{\alpha \beta \gamma \delta}$ satisfying $\epsilon^{0123}=+1$, whereas the Levi-Civita tensor is given by $\hat{\epsilon}^{\alpha \beta \gamma \delta}=\frac{1}{\sqrt{-g}} \epsilon^{\alpha \beta \gamma \delta}$.

\section{Chiral gravitational effect in a dynamical background}

We first discuss the gravitational parity-violating effect in a chiral asymmetric fermion background, which is referred as the chiral gravitational effect in ref. [48], focusing on the Minkowski spacetime background for simplicity. In order to consider this phenomenon in a cosmological setup, e.g. primordial thermal plasma of the Universe, one needs to incorporate the dynamical behavior of the chiral fermion background, associated with its generation mechanism and the chirality-flipping interactions. We will see that the dilution due to the cosmic expansion also affects the dynamics of gravitons when we consider the generalization to the FRW background. Such a dynamics has been taken into account in ref. [69] where the effect of the cosmic neutrino background on the propagating gravitational waves through the induced gravitational Chern-Simons term is considered. We here give a brief review of the formulation in ref. [69] (see also ref. [82]), which is consistent with ref. [64], and re-examine it in the context of chiral gravitational effect for the application to cosmology.

Let us consider the tensor perturbation (graviton) around the Minkowski spacetime $g_{\mu \nu} \simeq \eta_{\mu \nu}+h_{\mu \nu}$ with a chiral asymmetric fermion background. The chiral asymmetry of the fermion system is characterised by the chiral chemical potential $\mu_{5}$ and is described by a quadratic term of fermions in the effective Lagrangian as

$$
S_{\mu}^{\mathrm{eff}}=\int d^{4} x \mu_{5} \bar{\psi} \gamma^{0} \gamma^{5} \psi
$$

Throughout this paper, we focus on the regime where the typical momentum of the graviton, $k$, is larger than the background temperature $T$ and the chemical potential $\mu_{5}$, namely, $k \gg\left|\mu_{5}\right|, T,{ }^{6}$ where the contribution from the induced Chern-Simons term dominates other

\footnotetext{
${ }^{6}$ We will clarify the reason why we need to assume such a parameter region before the end of this section.
} 


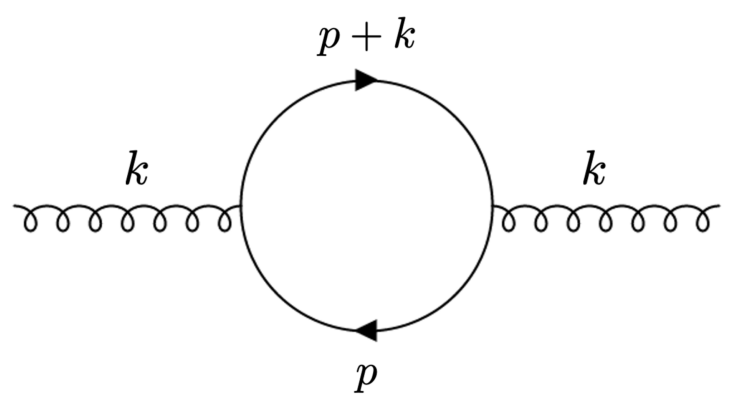

Figure 1. 1-loop correction to the graviton propagator relevant to the induction of the gravitational Chern-Simons term.

contributions, e.g. non-local thermal corrections, found in refs. $[48,64]$ in the anomalous responses. The term induced by the chiral asymmetry (eq. (2.1)) modifies the propagator of the fermion, which in turn gives the parity-violating contribution to the graviton polarization tensor $\Pi_{\mu \nu \rho \sigma}$ through the loop correction as depicted in figure 1. In the massless limit of the fermion $m \ll k$, the leading parity-violating correction to the polarization tensor from the fermion loop takes the following simple form in the momentum space [69]:

$$
\Pi_{\mu \nu \rho \sigma}^{\mathrm{PV}}=\mathrm{i} \frac{\mu_{5}}{192 \pi^{2}}\left\{\epsilon_{\mu \rho \alpha 0} k^{\alpha}\left[k_{\nu} k_{\sigma}-k^{2} \eta_{\nu \sigma}\right]+(\mu \leftrightarrow \nu)+(\rho \leftrightarrow \sigma)+(\mu \leftrightarrow \nu, \rho \leftrightarrow \sigma)\right\},
$$

where $k$ denotes the momentum of the graviton. Here the higher order terms in $\mu_{5}$ and $k$ are neglected. This loop correction can be rewritten in terms of the gravitational ChernSimons term in the effective action up to the second order in $h_{\mu \nu}$ as

$$
\begin{aligned}
S_{2}^{\mathrm{eff}, \mathrm{PV}} & =-\frac{\mu_{5}}{192 \pi^{2}} \int d^{4} x \epsilon_{\mu \rho \alpha 0} h^{\mu \nu} \partial^{\alpha}\left(\square h^{\rho \sigma} \eta_{\nu \sigma}-\partial_{\nu} \partial_{\sigma} h^{\rho \sigma}\right) \\
& =\frac{\mu_{5}}{96 \pi^{2}} \int d^{4} x K^{0},
\end{aligned}
$$

where $K^{0}$ is the linearized 0-th component of the four dimensional Chern-Simons topological current, $K^{\mu} \equiv \epsilon^{\mu \nu \rho \sigma} \Gamma_{\nu \beta}^{\alpha}\left(\partial_{\rho} \Gamma_{\sigma \alpha}^{\beta}+(2 / 3) \Gamma_{\rho \gamma}^{\beta} \Gamma_{\sigma \alpha}^{\gamma}\right)$.

It is useful to introduce a spurious scalar field $\theta$ which is related to the chiral chemical potential as

$$
\partial_{\mu} \theta=\left(\frac{\mu_{5}}{192 \pi^{2}}, 0,0,0\right)
$$

By virtue of this field, the influence of the general background with the chiral asymmetry results in the form of gravitational Chern-Simons term as

$$
S_{\mathrm{eff}}^{\mathrm{PV}}=\int d^{4} x\left(\partial_{\mu} \theta\right) 2 K^{\mu}=-\int d^{4} x \sqrt{-g} \theta R \tilde{R},
$$

with the Chern-Pontryagin density being defined as

$$
R \tilde{R} \equiv R_{\mu \nu \alpha \beta} \tilde{R}^{\mu \nu \alpha \beta}=-\frac{1}{2} \frac{\epsilon^{\alpha \beta \gamma \delta}}{\sqrt{-g}} R_{\alpha \beta \rho \sigma} R_{\gamma \delta \mu \nu} g^{\mu \rho} g^{\nu \sigma} .
$$


Note that here the induced parity-violating term is extended into the covariant expression. Although the derivation of the effective action is based on the constant chemical potential background in the Minkowski spacetime, the covariant expression enables us to apply the effective action to more general situations where the chemical potential effectively has nontrivial time dependence and to the curved spacetime case such as the FRW spacetime. Such an extension would be reasonable since our approach can be understood as the effective field theory where the (spacetime) symmetry of the theory is spontaneously broken by the spurious field $\theta$. See also the related studies of the CME $[67,68]$, which also introduced the spurious axion field $\theta(t, \vec{x})$ coupled to the $F \tilde{F}$ term in order to represent the asymmetric state of the thermal plasma. ${ }^{7}$

Let us clarify the physical property of the induced Chern-Simons term by expanding the effective action around the Minkowski spacetime background. After taking the transverse and traceless gauge, the action can be expanded up to the second order in the metric perturbation $h_{i j}$ as

$$
\begin{aligned}
& S_{\mathrm{E}-\mathrm{H}}+S_{\mathrm{eff}}^{\mathrm{PV}} \simeq \frac{M_{\mathrm{Pl}}^{2}}{8} \int d^{4} x {\left[\left\{\dot{h}_{j}^{i} \dot{h}^{j}{ }_{i}-\left(\partial_{k} h^{i}{ }_{j}\right)\left(\partial^{k} h^{j}{ }_{i}\right)\right\}\right.} \\
&\left.+\left(\frac{8 \dot{\theta}}{M_{\mathrm{Pl}}^{2}}\right) \epsilon^{0 i j k}\left\{\dot{h}^{q}{ }_{i} \partial_{j} \dot{h}_{k q}-\left(\partial^{r} h^{q}{ }_{i}\right) \partial_{j} \partial_{r} h_{k q}\right\}\right],
\end{aligned}
$$

where $S_{\mathrm{E}-\mathrm{H}}$ denotes the Einstein-Hilbert term and the dot denotes the time derivative. This action leads to the equation of motion

$$
\begin{aligned}
\ddot{h}_{i}^{j}-\nabla^{2} h_{i}^{j} & =-\frac{8}{M_{\mathrm{Pl}}^{2}} \epsilon^{j m n} \partial_{m}\left(\ddot{\theta} \ddot{h}_{i n}+\ddot{\theta} \dot{h}_{i n}-\dot{\theta} \nabla^{2} h_{i n}\right) \\
& =-\frac{\epsilon^{j m n}}{24 \pi^{2} M_{\mathrm{Pl}}^{2}} \partial_{m}\left(\mu_{5} \ddot{h}_{i n}+\dot{\mu}_{5} \dot{h}_{i n}-\mu_{5} \nabla^{2} h_{i n}\right),
\end{aligned}
$$

where we have used eq. (2.4) in the second line. We will study its consequences in the following sections.

Before studying the solutions of eq. (2.8), let us investigate the physical implications of the induced term. The modification of the equation of motion in eq. (2.8) reflects the fact that the Chern-Simons term in the effective action leads to the transverse-traceless part of the induced parity-violating energy momentum tensor in the asymmetric fermion background as

$$
T^{(\text {ind })}{ }_{i} \simeq 4 \epsilon^{j m n}\left\{\dot{\theta} \partial_{m}\left(\partial_{t}^{2}-\nabla^{2}\right)+\ddot{\theta} \partial_{m} \partial_{t}\right\} h_{i n} .
$$

From this expression, one can understand that the effect is analogous to the CME where the electric current is induced by the external magnetic field. In the present case, the external gravitational field applied to the chiral asymmetric system induces the energy momentum tensor, which is the "current" for the diffeomorphism. Therefore, it is reasonable to call this phenomenon as the chiral gravitational effect.

The chiral gravitational effect in a static thermal background has been studied in ref. [48], where the authors particularly focused on the anomalous corrections to the dis-

\footnotetext{
${ }^{7}$ The "spurious" scalar field $\theta$ actually appears as a collective excitation in the anomalous hydrodynamics. See ref. [83] for the classical case and ref. [84] for the quantum case.
} 
persion relation and studied the properties of the corrections in various limits of parameters. The analysis is based on the gravitational response function derived in literature [64, 65]. The term proportional to $\mu_{5}$ in eq. (2.9) actually coincides with the one derived in the previous study [64] in the static limit of the gravitational field. On the other hand, the latter term proportional to $\dot{\mu}_{5}$ appears only when we introduce the effective action with the induced Chern-Simons term (eq. (2.5)) that allows the time-dependent chiral chemical potential. In this way, the dynamical behavior of the chiral fermion background has been incorporated into the chiral gravitational effect. Although we here demonstrate it in the Minkowski spacetime case, a curved background geometry is also expected to give nontrivial contributions to the chiral gravitational effect due to the non-conformal nature of the graviton as we will see in section 4 .

We would also like to mention the difficulty in the Lagrangian description of the thermal correction as well as the higher order corrections from the non-zero chemical potential. The thermal and chemical potential corrections to the two point function found in refs. [64, 65] contain non-local contributions as well as local one. The latter corresponding to the gravitational Chern-Simons term can be written in a covariant form as we have seen. On the other hand, it is in general difficult to rewrite the former in such a form. Indeed, in ref. [82], the local as well as non-local Lagrangian terms including thermal corrections are shown at the leading order of $\mu_{5}$ in a non-covariant form, but a covariant Lagrangian description that takes into account all the contributions found in refs. [64, 65] has not been obtained, if ever. The covariant (local) Lagrangian is an important guiding principle for us to extend the effective Lagrangian (2.5) to that in more general backgrounds. It is desirable if we would have a covariant form of the non-local contributions to apply such a prescription, but at present we have not reached at it yet. This is the reason why we discuss the parameter region $k \gg T,\left|\mu_{5}\right|$ so that the local contribution dominates over non-local ones. We should also emphasize that even in a different parameter region, where non-local terms dominate the graviton dynamics, the local contribution is present and the effects we discuss in this paper would remain.

Before closing this section, we would like to comment on the possible issues associated with the gravitational anomaly term (eq. (2.7)). As we can see from eq. (2.8), the additional term contributes to the kinetic term (or the dispersion relation) if we Fourier-transform the expression. In particular, such corrections depend on the momentum. Since this contribution to two point function is positive or negative depending on the chirality, either left or right modes with large enough momentum $k$ may become ghost-like. ${ }^{8}$ Therefore, one should introduce a cut-off scale to the theory above which the effective theory is broken down. In our following analysis, we will treat the correction perturbatively, or equivalently, we consider the energy and momentum range to be much below the cut-off scale, which is reasonable to avoid the appearance of the ghost-like mode mentioned above. ${ }^{9}$

\footnotetext{
${ }^{8}$ This issue is one of the obstacles in gravitational leptogenesis with the gravitational anomaly term, see e.g. ref. [55].

${ }^{9}$ Our treatment of the correction is different from that in ref. [69], where the authors discuss the constraint e.g. on the photon production caused by the ghost-like graviton. In order to avoid the subtlety associated with the treatment of such unstable modes, we simply consider the momentum range where the kinetic terms of both the left and right graviton modes are positive.
} 


\section{Gravitational birefringence and the backreaction to chiral plasma in the Minkowski background}

Now we are ready to investigate how the induced energy momentum tensor contributes to the propagation of gravitational waves. For this purpose, in section 3.1, we shall solve the equation of motion for gravitons by taking the gravitational Chern-Simons term as perturbation in the Minkowski spacetime. We will see that $\dot{\mu}_{5}$ dependence in eq. (2.9), which is absent in the case of the CME, causes the gravitational birefringence. With the corrected gravitational wave solutions, we also analyze the anomaly equation in order to take into account the backreaction of gravitational waves to chiral plasma in section 3.2. Such a comprehensive study of this system reveals the nontrivial interplay between gravitational waves and chiral plasma as we will present.

\subsection{Propagation of gravitational waves}

We start with a toy model in the Minkowski spacetime background. Specifically, we consider gravitational waves propagating in the $z$-direction throughout this section. Let us define the circular polarization tensors for the right and left-handed modes as

$$
p_{i j}^{A} \equiv \frac{1}{\sqrt{2}}\left(p_{i j}^{+}+\mathrm{i} \lambda_{A} p_{i j}^{\times}\right)=\frac{1}{\sqrt{2}}\left(\begin{array}{ccc}
1 & \mathrm{i} \lambda_{A} & 0 \\
\mathrm{i} \lambda_{A} & -1 & 0 \\
0 & 0 & 0
\end{array}\right), \quad \text { with } \quad \lambda_{\mathrm{R}}=+1, \lambda_{\mathrm{L}}=-1,
$$

which satisfy the following relations:

$$
\begin{aligned}
p_{i j}^{\mathrm{R}} p^{i j \mathrm{R}} & =p_{i j}^{\mathrm{L}} p^{i j \mathrm{~L}}=0, \\
p_{i j}^{\mathrm{R}} p^{i j \mathrm{~L}} & =2, \\
\epsilon^{m 3 j} p_{i j}^{A} & =-\mathrm{i} \lambda_{A} p^{m}{ }_{i}{ }^{A} .
\end{aligned}
$$

Expanding the metric perturbation as $h_{i j}=\sum_{A=R, L} h_{A}(t, z) p_{i j}^{A}$, we can rewrite the equation of motion (2.8) as

$$
\left(\partial_{t}^{2}-\partial_{z}^{2}\right) h_{A}(t, z)=\frac{\mathrm{i} \lambda_{A}}{24 \pi^{2} M_{\mathrm{Pl}}^{2}} \partial_{z}\left(\mu_{5} \ddot{h}_{A}(t, z)+\dot{\mu}_{5} \dot{h}_{A}(t, z)-\mu_{5} \partial_{z}^{2} h_{A}(t, z)\right),
$$

so that the right- and left-handed modes are decoupled from each other. Here $h_{L}(t, z)$ and $h_{R}(t, z)$ are related to each other as $h_{\mathrm{R}}(t, z)=h_{\mathrm{L}}^{*}(t, z)$, which follows from the reality condition.

Let us investigate the correction to the plane wave solution of the form $h_{A}(t, z)=$ $h_{A}(t) e^{\mathrm{i} k z}+h_{\bar{A}}^{*}(t) e^{-\mathrm{i} k z}$, where $\bar{A}$ is defined as $\overline{\mathrm{R}}=\mathrm{L}$ and $\overline{\mathrm{L}}=\mathrm{R}$. From eq. (3.3), $h_{A}(t)$ should satisfy

$$
\begin{aligned}
\left(\frac{d^{2}}{d t^{2}}+k^{2}\right) h_{A}(t) & =-\frac{\lambda_{A} k}{24 \pi^{2} M_{\mathrm{Pl}}^{2}}\left\{\mu_{5}\left(\frac{d^{2}}{d t^{2}}+k^{2}\right)+\dot{\mu}_{5} \frac{d}{d t}\right\} h_{A}(t) \\
& \equiv-\frac{\lambda_{A} k}{24 \pi^{2} M_{\mathrm{Pl}}^{2}} \hat{F}_{k}(t) h_{A}(t) .
\end{aligned}
$$


One can see that the first two terms in the right-hand side of eq. (3.4), coming from the induced Chern-Simons term, is proportional to its left-hand side originally contained in the Einstein-Hilbert term. This suggests that the Chern-Simons term may lead to the strong coupling and the ghost instability for the graviton when the effective coupling constant, $k\left|\mu_{5}\right| / 24 \pi^{2} M_{\mathrm{Pl}}^{2}$, is too large, as we mentioned in the previous section. Such an instability is inevitable for large momentum and we cannot predict the dynamics of the system at the scale higher than the emergent strong coupling scale, $k>24 \pi^{2} M_{\mathrm{Pl}}^{2} /\left|\mu_{5}\right|$. This instability is harmless if

$$
\left|\mu_{5}\right| \ll 24 \pi^{2} M_{\mathrm{Pl}}
$$

is satisfied since the positivity of the coefficient of $d^{2} / d t^{2}$ in eq. (3.4) is ensured and the emergent scale lies in the strong coupling scale of the Einstein-Hilbert action, that is, the Planck scale $M_{\mathrm{Pl}}$. We shall take eq. (3.5) as a conservative constraint on the chiral chemical potential.

Under such an assumption on the parameter space, we can safely treat the contribution of the Chern-Simons term in the right-hand side of eq. (3.4) as a perturbation. We incorporate the effect of the gravitational Chern-Simons term perturbatively by using the Green function method. Here, the Green function is defined as the solution to

$$
\left(\partial_{t}^{2}+k^{2}\right) G\left(t, t^{\prime}\right)=\delta\left(t-t^{\prime}\right)
$$

The zero-th order solution $h_{A}^{(0)}(t)$ is nothing but the solution to eq. (3.4) for $\mu_{5}=\mu_{5}=0$. Then, the first order correction to the zero-th order solution can be evaluated as

$$
h_{A}^{(1)}(t)=h_{A}^{(0)}(t)-\lambda_{A} \frac{k}{24 \pi^{2} M_{\mathrm{Pl}}^{2}} \int_{-\infty}^{\infty} d t^{\prime} G\left(t, t^{\prime}\right) \hat{F}_{k}\left(t^{\prime}\right)\left(h_{A}^{(0)}\left(t^{\prime}\right)\right)
$$

where $G\left(t, t^{\prime}\right)=\Theta\left(t-t^{\prime}\right) \sin k\left(t-t^{\prime}\right) / k$ is the explicit form of the Green function in this case with $\Theta(t)$ denoting the Heaviside step function. If we take the zero-th order plane wave solution to be that for the left-handed polarization mode, $h_{\mathrm{L}}^{(0)}(t)=A e^{-\mathrm{i} k t}$, eq. (3.7) reads

$$
h_{\mathrm{L}}^{(1)}(t)=A\left(1+\frac{k}{48 \pi^{2} M_{\mathrm{Pl}}^{2}} \Delta \mu_{5}(t)\right) e^{-\mathrm{i} k t}-\frac{A k}{48 \pi^{2} M_{\mathrm{Pl}}^{2}}\left(\int_{-\infty}^{t} d t^{\prime} \dot{\mu}_{5}\left(t^{\prime}\right) e^{-2 \mathrm{i} k t^{\prime}}\right) e^{\mathrm{i} k t},
$$

where $\Delta \mu_{5}(t)=\mu_{5}(t)-\mu_{5}(-\infty)$. As one can see from eq. (3.8), gravitational waves feel the time variation of the chiral chemical potential during the propagation but not the non-vanishing chiral chemical potential itself. If $\dot{\mu}_{5}>0$ for a while, as an example, the positive frequency mode is amplified and the negative frequency mode is simultaneously generated for the left-handed polarization. Note that the anomaly corrected solution for the right-handed polarization mode with a positive frequency, $h_{\mathrm{R}}(t)=A e^{-\mathrm{i} k t}$, is obtained by replacing $\mu_{5}$ to $-\mu_{5}$ in eq. (3.8) and results in the opposite correction. From these observations, we conclude that the chiral gravitational effect causes the time variation of the helicity, or the gravitational birefringence. In what follows we mainly focus on the case where only the left-handed polarization mode exists, but the generalization to the situation where both circular polarization modes exist can be done in a straightforward way. 
We find a remarkable feature of the chiral gravitational effect. As read from eq. (3.8), the correction in the first term can vanish regardless of the evolution history of $\mu_{5}$ if the chiral imbalance today is the same as its initial value since it is proportional to the net chemical potential difference between the initial and final values, $\Delta \mu_{5}$. On the other hand, the second term is in general non-vanishing even if $\Delta \mu_{5}$ eventually vanishes, thanks to the nontrivial phase factor $e^{-2 \mathrm{i} k t}$ in the integrand and depends on the evolution history of $\mu_{5}$. This implies that the chiral gravitational effect in a time-dependent background leaves the "memory" of the chiral imbalance in the time evolution history. Since in the early Universe, nontrivial time evolution of $\mu_{5}$ can be realized by chirality-violating processes such as the GUT baryogenesis as well as the chirality flip by the Yukawa interactions, gravitational birefringence may take place in the primordial chiral plasma. In such a situation, the primordial gravitational wave can be a probe to investigate the dynamical chirality-violating processes in the early Universe through the chiral gravitational effect. This property also appears in the expanding background as we will see later. However, one needs to care the possibility that the backreaction to the chiral plasma from gravitational waves might affect the memory of such processes. In the next subsection, we analyze the anomaly equation to quantify the backreaction and show that their effect on the memory of the gravitational waves is negligibly small.

We should emphasize that the existence of non-zero $\mu_{5}$ at the beginning does not affect the propagation of gravitational waves through the local contribution represented by the Chern-Simons term. This behavior is quite different from the electromagnetic case where the chiral plasma instability is caused by the non-zero chemical potential itself but not its time variation [30,31]. This different dependence on $\mu_{5}$ can be ascribed to the spin structure of the field of interest. Technically, the gravitational Chern-Simons term, which involves the spin 2 field, contains more derivatives than that for the spin 1 gauge field. As a result, $\ddot{\theta} \sim \dot{\mu}_{5}$ appears in the first order in $\mu_{5}$. Physically speaking, the structure of the effective action of a spin $n \geq 1$ field is determined by the gauge symmetry associated with the field. For graviton, the diffeomorphism determines a possible form of the effective action and as a result, it becomes relevant only when the chemical potential is time-dependent. As we quoted in section 2, however, there are non-local contributions to the graviton dispersion relation proportional to $\mu_{5}^{3}$ and $T^{2} \mu_{5}[64,65,82]$, which cannot be treated in our local effective Lagrangian. ${ }^{10}$ If such non-local effects dominate the graviton propagation, a constant chemical potential can modify the gravitational wave dynamics as shown in ref. [48].

\subsection{Backreaction to chiral plasma from gravitational waves}

We have obtained the anomaly corrected gravitational wave solution and found that the time variation of the chiral chemical potential can cause the gravitational birefringence. In order to comprehensively trace the evolution of this system, we must investigate the backreaction from the gravitational waves to the chiral plasma. To our best knowledge,

\footnotetext{
${ }^{10}$ In other words, the absence of the chiral effects in the graviton propagation for $\dot{\mu}_{5}=0$ would be independent of our perturbative treatment of $\mu_{5}$ as can be read from eq. (3.4).
} 
such an issue has not been addressed so far. For this purpose, we here analyze the anomaly equation to investigate the backreaction by using the wave solutions obtained in the previous section.

Gravitational anomaly expresses the violation of the chiral charge conservation of the fermion due to the presence of non-vanishing topological configuration of the gravitational wave as

$$
\nabla_{\mu} j_{5}^{\mu}=-\frac{1}{12(4 \pi)^{2}} R \tilde{R} .
$$

What physically happens is that the chiral asymmetry of gravitational waves acts as a biased background for the evolution of the Dirac equation [50]. Let us evaluate the contribution of the right-hand side of the anomaly equation (3.9) with the gravitational wave solution obtained in section 3.1. In the Minkowski spacetime, the spatial average of $R \tilde{R}$ can be expanded as

$$
\begin{aligned}
\langle R \tilde{R}\rangle & \simeq \partial_{t}\left\{\epsilon^{i j k}\left(\dot{h}_{i l} \partial_{j} \dot{h}_{k}{ }^{l}-\partial_{l} h_{i m} \partial^{l} \partial_{j} h_{k}{ }^{m}\right)\right\} \\
& =4 \partial_{t}\left\{k\left(\left|\dot{h}_{\mathrm{R}}(t)\right|^{2}-\left|\dot{h}_{\mathrm{L}}(t)\right|^{2}\right)-k^{3}\left(\left|h_{\mathrm{R}}(t)\right|^{2}-\left|h_{\mathrm{L}}(t)\right|^{2}\right)\right\} .
\end{aligned}
$$

By substituting eq. (3.8) into eq. (3.10), we obtain the evolution equation for the chiral charge carried by fermions with the gravitational anomaly from the plane wave solution for the left-handed polarization mode, $h_{\mathrm{L}}^{(0)}(t, z)=A e^{-i k(t-z)}$ or $h_{\mathrm{L}}^{(0)}(t)=A e^{i k t}, h_{\mathrm{R}}^{(0)}(t)=0 \operatorname{as}^{11}$

$$
\begin{aligned}
\dot{n}_{5} & =C \frac{A^{2} k^{4}}{3 \pi^{2} M_{\mathrm{Pl}}^{2}} \partial_{t}\left\{\int_{-\infty}^{t} d t^{\prime} \dot{\mu}_{5}\left(t^{\prime}\right) \cos 2 k\left(t-t^{\prime}\right)\right\}\left(1+\mathcal{O}\left(\frac{k \mu_{5}}{M_{\mathrm{Pl}}^{2}}, \frac{\dot{\mu}_{5}}{M_{\mathrm{Pl}}^{2}}\right)\right) \\
& =C \frac{A^{2} k^{4}}{3 \pi^{2} M_{\mathrm{Pl}}^{2}}\left\{\dot{\mu}_{5}(t)-2 k \int_{-\infty}^{t} d t^{\prime} \dot{\mu}_{5}\left(t^{\prime}\right) \sin 2 k\left(t-t^{\prime}\right)\right\}\left(1+\mathcal{O}\left(\frac{k \mu_{5}}{M_{\mathrm{Pl}}^{2}}, \frac{\dot{\mu}_{5}}{M_{\mathrm{Pl}}^{2}}\right)\right),
\end{aligned}
$$

where $n_{5}=j_{5}^{0}$ and $C \equiv 1 / 12(4 \pi)^{2}$. Here we assume that the spatial gradient of the chiral charge is always negligibly small and also neglect the contributions from the spatial component of the chiral current. From eq. (3.11), one can find that the "modulated" chemical potential results in the violation of the chiral charge in the fermion sector, which in turn affects the chemical potential.

When the plasma is thermalized, chiral charge density is related to the chiral chemical potential as $n_{5} \simeq \mu_{5} T^{2} / 6$ for $\mu_{5} \ll T$. Assuming that the chirality-changing process is sufficiently slow and the equilibrium is always maintained, the variation of the charge can be written in terms of the chemical potential. As a simple example, let us first consider the case where the non-zero constant $\mu_{5}$ exists but there are no other dynamical chiral charge violating processes. In this case, the evolution of $\mu_{5}$ is determined merely by the anomaly induced backreaction (eq. (3.11)). Then we can write the kinetic equation of $\mu_{5}$

\footnotetext{
${ }^{11}$ If one consider the initially non-helical plane wave as $h_{\mathrm{L}}^{(0)}(t)=h_{\mathrm{R}}^{(0)}(t)=A e^{i k t}$, the right-handed polarization mode adds the same contribution as the left-handed polarization mode to the right-hand side of eq. (3.11). Thus, for the non-helical initial condition, one should replace $C=1 / 12(4 \pi)^{2}$ with $\tilde{C} \equiv 2 \times 1 / 12(4 \pi)^{2}$ in eq. (3.11).
} 
in the following form:

$$
\begin{aligned}
\dot{\mu}_{5}(t) & =\delta \partial_{t}\left\{\int_{-\infty}^{t} d t^{\prime} \dot{\mu}_{5}\left(t^{\prime}\right) \cos 2 k\left(t-t^{\prime}\right)\right\} \\
& =\delta\left\{\dot{\mu}_{5}(t)-2 k \int_{-\infty}^{t} d t^{\prime} \dot{\mu}_{5}\left(t^{\prime}\right) \sin 2 k\left(t-t^{\prime}\right)\right\},
\end{aligned}
$$

where $\delta \equiv \frac{2 C}{\pi^{2}} A^{2} \frac{k^{4}}{T^{2} M_{\mathrm{Pl}}^{2}}$ which characterizes the strength of the backreaction. By substituting the integrated form of eq. (3.12) into its differentiated form, one can obtain the following second-order differential equation,

$$
(1-\delta) \Delta \ddot{\mu}_{5}+4 k^{2} \Delta \mu_{5}=0
$$

Hereafter we consider the case $\delta \ll 1$, which is realized with sufficiently small $A$ and $k .^{12}$ Clearly this equation describes the harmonic oscillator. However, eq. (3.12) gives the initial condition $\Delta \dot{\mu}_{5}=0$ which results in the trivial solution $\Delta \mu_{5}(t)=0$. As we have emphasized in the previous section, this behavior is completely different from the CME where the instability is triggered just by the existence of the non-zero chemical potential through the local anomalous contribution. ${ }^{13}$

Next, we consider the case where the non-trivial time evolution of $\mu_{5}$ is sourced by a chirality-inducing process, which plays the role of an external force to the static harmonic oscillator. The backreaction of the chiral gravitational effect (3.11) is expected to perturb this dynamics slightly. In this situation, we can expand the chiral chemical potential in the following way:

$$
\mu_{5}(t)=\mu_{5}^{(0)}(t)+\mu_{5}^{(1)}(t),
$$

where the first term represents the chirality generation governed by the dominant process and the second term describes the perturbative backreaction due to the anomaly equation (eq. (3.11)). Having in mind the decay of heavy particles, such as heavy colored Higgs field $H^{c} \subset \mathbf{5}$ in the case of SU(5) GUT baryogenesis [33, 47], we consider a model where $\mu_{5}^{(0)}(t)$ is given as

$$
\dot{\mu}_{5}^{(0)}(t)= \begin{cases}0, & \text { for } \quad t<0 \\ \Gamma \exp (-t / \tau), & \text { for } \quad t \geq 0\end{cases}
$$

where the decay of the heavy particle turns on at $t=0$ with $\tau$ being the lifetime of the heavy particle. $\mu_{5}^{(1)}(t)$ then can be evaluated perturbatively in powers of the parameter $\delta$ which characterizes the strength of the backreaction as

$$
\dot{\mu}_{5}^{(1)}(t)=\delta\left\{\dot{\mu}_{5}^{(0)}(t)-2 k \int_{0}^{t} d t^{\prime} \dot{\mu}_{5}^{(0)}\left(t^{\prime}\right) \sin 2 k\left(t-t^{\prime}\right)\right\}+\mathcal{O}\left(\delta^{2}\right),
$$

\footnotetext{
${ }^{12}$ We however assume that $k$ is not too small so that the conditions for the non-local effects to be negligibly small, $\mu_{5}, T \ll k$, are satisfied.

${ }^{13}$ This point also shows the difference between $\mathrm{CME}$ and the chiral gravitational effect, as the constant chemical potential causes non-trivial evolution of the system for the former through the local Lagrangian term but for the latter only through the non-local contributions.
} 
where the right-hand side represents the contribution from the anomaly as in eq. (3.12). Using eqs. (3.15) and (3.16) with the initial condition $\Delta \mu_{5}(0)=0, \mu_{5}(t)$ can be expanded as

$$
\begin{aligned}
\Delta \mu_{5}(t) & =\int_{-\infty}^{t} d t^{\prime}\left(\dot{\mu}_{5}^{(0)}\left(t^{\prime}\right)+\dot{\mu}_{5}^{(1)}\left(t^{\prime}\right)\right) \\
& \simeq \Gamma \tau\left(1-e^{-\frac{t}{\tau}}\right)-\frac{\Gamma \tau \delta}{1+4 k^{2} \tau^{2}}\left(\cos (2 k t)+2 k \tau \sin (2 k t)-e^{-\frac{t}{\tau}}\right)+\mathcal{O}\left(\delta^{2}\right) .
\end{aligned}
$$

The first term is nothing but the leading contribution from the source term whereas the second term represents the backreaction from gravitational waves due to the gravitational anomaly. The latter is significantly suppressed at least by a factor of $\delta$, but remains even in the late time where the source contribution eventually disappears. Compared to the previous discussion, here a non-zero initial $\dot{\mu}_{5}$ is allowed with the help of the non-vanishing source term at an earlier time. Therefore a non-zero oscillatory term, which is a solution to eq. (3.13) with $\delta=0$, appears as the consequence of the backreaction. Thus, one can conclude that the oscillatory conversion between the chirality of the plasma and the helicity of the gravitational waves is triggered by the source.

By investigating the structure of eq. (3.17) in more depth, we can read off the following general features which appear when one incorporates the external chirality-changing process into the system, which eventually disappears at a late time. At an early time, the backreaction $\left(\dot{\mu}_{5}^{(1)}\right)$ is suppressed by $\delta$ and the dynamics of $\mu_{5}(t)$ is determined by the source. As discussed in the previous subsection, $\dot{\mu}_{5}^{(1)}$ contains the contribution from the anomaly induced correction of the gravitational waves in eq. (3.8), which includes the time integration of $\dot{\mu}_{5} e^{-2 \mathrm{i} k t}$. This is what we call the "memory effect". If the magnitude of $\dot{\mu}_{5}^{(0)}$ monotonically decays as in this model, the correction to the gravitational wave propagation is dominated by the "memory" of the source dynamics in early times. Then eventually the kinetic equation for the chiral chemical potential is dominated by the contributions from the memory effect. In the right hand side of eq. (3.16), for example, it corresponds to the contribution from the second term in the parentheses. By performing the integral over the early times, the memory contribution can be approximated as $\propto \delta k C \sin (2 k t+\alpha)$. The factor $C$ denotes the result of the integral and is dominated by the early time contribution as $C \sim \dot{\mu}_{\text {ini }} \Delta t$, where $\Delta t$ is the time scale of the decay of the source. One can see that this contribution remains as the oscillating source even in the late time. Thus we can understand that the non-decaying oscillation in the second term of eq. (3.17) originates from this early memory contributions. ${ }^{14}$ Nevertheless, when the source contribution becomes comparable to the backreaction, we can no longer treat the source $\dot{\mu}_{5}^{(0)}$ and backreaction $\dot{\mu}_{5}^{(1)}$ separately. This means that there should be a non-trivial interplay between source and the backreaction in much later time. Does such a regime affect the gravitational wave behavior? The answer is negative. Although the interplay between the source and backreaction in the late time can be non-trivial, their amplitude is expected to be suppressed at least by a factor of $\delta$. Since the memory, or the helicity accumulated in gravitational waves, consists of the dominant source contribution in the early time, such a late time contribu-

\footnotetext{
${ }^{14}$ For much later time, $t \gg \Delta t / \delta$, the integrated late time contributions of the source might become comparable to the early time contributions in general.
} 
tion should be negligible for the modifications of the gravitational wave propagation. From these observations, we expect that in general the leading contribution in the backreaction comes from the early time memory effect. However, the late time chemical potential would be "sourced" by the early time memory effect, which may lead to nontrivial dynamics of the chemical potential.

To show the generality of the discussion in the above, let us investigate the effect of another example, the chirality-flipping interactions, which is responsible for the decay of the chiral asymmetry. Note that the chirality is not an exact symmetry of the Standard Model of particle physics and is flipped by Yukawa interactions. To mimic such chirality-flipping interactions, we consider the following term,

$$
\dot{\mu}_{5}(t) \ni-\Gamma_{f} \mu_{5}(t), \quad \text { for } \quad t \geq 0,
$$

with non-vanishing initial chiral asymmetry, $\mu_{5}^{\text {ini }} \neq 0$ at $t=0$. Here $\Gamma_{f}$ is the chiralityflipping rate, which turns on at $t=0$. The anomaly-corrected kinetic equation for the chiral chemical potential is then given by

$$
\dot{\mu}_{5}(t)=-\Gamma_{f} \mu_{5}(t)+\delta\left\{\dot{\mu}_{5}(t)-2 k \int_{0}^{t} d t^{\prime} \dot{\mu}_{5}\left(t^{\prime}\right) \sin 2 k\left(t-t^{\prime}\right)\right\} .
$$

At an early time where the backreaction can be treated perturbatively, the dynamics is dominated by the chirality-flipping effect and $\mu_{5}(t) \sim \mu_{5}^{\text {ini }} e^{-\Gamma_{f} t}$, similar to the chirality inducing process. As discussed in the above for that case, we expect that the dominant memory effect on gravitational waves originates from this early stage. In the later time, the chemical potential exponentially decays and eventually the backreaction due to the early memory effect becomes comparable to the leading contribution. Then, one needs to solve eq. (3.19) without a perturbative method in $\delta$. Practically, it is useful to replace the integro-differential equation (3.19) with a differential equation. In a similar way how we obtained eq. (3.13), eq. (3.19) can be rewritten as

$$
(1-\delta) \dddot{\mu}_{5}(t)+\Gamma_{f} \ddot{\mu}_{5}(t)+4 k^{2} \dot{\mu}_{5}(t)+4 k^{2} \Gamma_{f} \mu_{5}(t)=0 .
$$

Note that the initial conditions for $\mu_{5}$ and its time derivatives at $t=0$ are fixed by eq. (3.19) and its differentiated form up to the second order derivative, namely, $\mu_{5}(0)=\mu_{5}^{\text {ini }}$, $\dot{\mu}_{5}(0) \simeq-\Gamma \mu_{5}^{\text {ini }}(1+\delta), \ddot{\mu}_{5}(0) \simeq \Gamma^{2} \mu_{5}^{\text {ini }}(1+2 \delta)$. Here we solve eq. (3.20) numerically for the parameters $\Gamma_{f} / k=1$, and $\delta=0.01$. Figure 2 shows the evolution of the chiral asymmetry in earlier times. We can see that the initial asymmetry is exponentially damped in proportion to $\exp [-\Gamma t]$ and a small slowly-decaying oscillation follows with $\propto \exp [-(2 / 5) \delta \Gamma t]$. This behavior can be understood as follows. By taking $\delta=0$, the formally zero-th order solution in $\delta$ can be obtained as

$$
\mu_{5}(t)=\mu_{5}^{\mathrm{I}} \exp \left[-\Gamma_{f} t\right]+\mu_{5}^{\mathrm{II}} \cos (2 k t)+\mu_{5}^{\mathrm{III}} \sin (2 k t) .
$$

While the first term represents the dominant exponentially damping contribution, the second and third contributions appear due to the backreaction and show the oscillatory behavior with the same frequency to the one in eq. (3.17). By considering the connection 


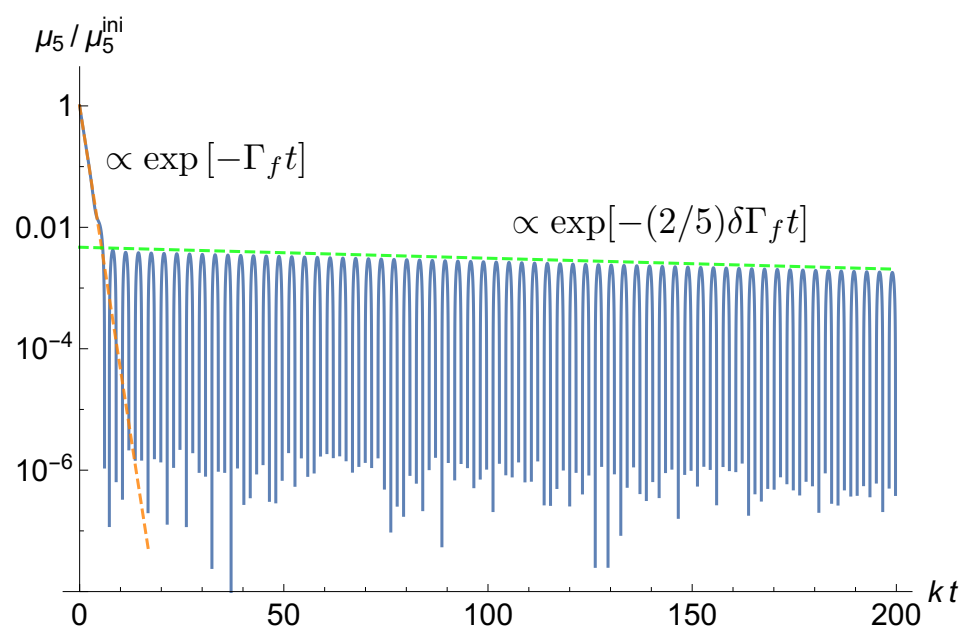

Figure 2. Numerical calculation of the solution to eq. (3.20) in the earlier times with $\Gamma_{f}=k$ and $\delta=0.01$. For $t \lesssim 1 / \Gamma_{f}$, the initial asymmetry is washout in a rate proportional to $\exp \left[-\Gamma_{f} t\right]$. For $t \gg 1 / \Gamma_{f}$, the backreaction dominates and the chiral chemical potential shows the slowly decaying oscillation in proportion to $\exp \left[-c \delta \Gamma_{f} t\right]$ with $c \simeq 2 / 5$ in this parameter set.

to the initial condition, one can see that $\mu_{5}^{\mathrm{II}}$ and $\mu_{5}^{\mathrm{III}}$ is the order of $\delta$. As a result, $\mu_{5}$ shows an exponential decay and a small oscillation around it in the early time, which is similar to the chirality inducing process. One can see that the oscillatory part decays slowly, which is due to the finite $\delta$ correction to eq. (3.21) from eq. (3.20). Such a correction should be in proportion to $\exp [-c \delta \Gamma t]$ so that the decay disappears if one takes $\delta \rightarrow 0$ limit. Here $c$ is a numerical factor, which found to be $2 / 5$ in this parameter choice. Note that such decay rates can be obtained by solving the characteristic equation of (3.20), especially for the specific limit of $\Gamma / k$. We would like to emphasize that this is consistent with the discussion in the above. Here the parenthesis in the kinetic equation (3.19), or the backreaction from the gravitational wave propagation, dominated by the memory effect in the early time, $\sim \delta k C_{f} \sin (2 k t+\alpha)$, now acts as the source term and is equilibrated to the chirality-flipping term. Here $C_{f}$ denotes the dominant contribution of the integral over the early times, and $\alpha$ denotes the phase shift due to the time-dependent $\dot{\mu}_{5}$. This leads to the slowly decaying oscillation of $\mu_{5}$.

As a concluding remark of this section, let us re-emphasize the memory effect on gravitational waves. As mentioned in the previous subsection, gravitational birefringence takes place according to the history of the evolution of $\dot{\mu}_{5}$ during the propagation. In both the chirality induction and flipping processes, we have seen that the dynamics of $\mu_{5}$ is determined by the source at an early time and the backreaction is generically negligible there. The dominant memory effect originates from this early stage. However, in a reasonable setup, eventually the external source for $\mu_{5}$ decays and becomes comparable to the backreaction coming from the early time memory effect. The interplay between various contributions to $\mu_{5}$ dynamics makes its behavior rather complicated, but eventually $\mu_{5}$ would behave in an oscillatory form forced by the oscillating source due to the backreaction at least for a dura- 
tion with a factor of $1 / \delta$. Note that the much later time behavior of the chemical potential, which is rather non-trivial, would have less significant magnitude than that of the early time, which is dominated by the external sources. Thus, we conclude that the gravitational waves memorize the dominant evolution of the chirality and the memory is hardly affected by the backreaction. It is quite interesting that the memory effect induced by dynamics of $\mu_{5}$ in early times affects (or dominates) the late time behavior of the chemical potential itself through the backreaction. As we will see in the next section, such a property of the memory effect does not change even in the FRW background case where the cosmological expansion also contributes to the birefringence through the chiral gravitational effect.

\section{Chiral gravitational effect in the expanding background}

As mentioned in section 2, the non-conformal nature of the graviton gives a non-trivial background spacetime dependence on the propagation of the gravitational waves through the chiral gravitational effect. Having in mind the application to the cosmology, we discuss the chiral gravitational effect in the FRW spacetime in this section. We will present how the cosmic expansion plays a role in the chiral gravitational effect as follows. We first calculate the wave solutions for the modified equation of motion in a general time-dependent chiral asymmetric fermion background in section 4.1. Then, we apply them to the case for the chiral chemical potential diluted by the cosmic expansion and evaluate the backreaction on the chiral chemical potential in section 4.2.

\subsection{Propagation of gravitational waves}

Here we discuss the anomaly corrected gravitational wave solution in the expanding Universe and demonstrate how the effect of the expanding background appears in the chiral gravitational effect. Let us consider the flat FRW background $d s^{2}=a(\eta)^{2}\left[-d \eta^{2}+d \boldsymbol{x}^{2}\right]$ where $\eta$ represents the conformal time. With the help of the covariant expression, we can again start from the effective action obtained in section 2 , namely $S_{\mathrm{E}-\mathrm{H}}+S_{\mathrm{eff}}^{\mathrm{PV}}=$ $\int d^{4} x \sqrt{-g}\left[\left(M_{\mathrm{Pl}}^{2} / 2\right) R-\theta R \tilde{R}\right]$. Note that the spurious scalar field should now be related to the chiral chemical potential as $\partial_{\eta} \theta=\left(\mu_{5} a(\eta) / 192 \pi^{2}, 0,0,0\right)$ since $\partial_{\mu} \theta$ acts as a conformally-invariant field, which should be constant in the absence of the chiralitychanging interactions.

In the Fourier space, one can expand metric perturbation in the circularly polarized basis as

$$
h_{i j}(\eta, \mathbf{x})=\frac{1}{(2 \pi)^{3}} \int d^{3} k \sum_{A=\mathrm{R}, \mathrm{L}} p_{i j}^{A}(\boldsymbol{k}) h_{\boldsymbol{k}}^{A}(\eta) e^{i \boldsymbol{k} \cdot \boldsymbol{x}},
$$

where the circular polarization tensors in the three-dimensional momentum space ${ }^{15}$ satisfy

\footnotetext{
${ }^{15}$ Here we have generalized the circular polarization tensor, which was introduced in section 3.1 for $\boldsymbol{k} \propto(0,0,1)$.
} 
the following equations,

$$
\begin{aligned}
p_{i j}^{\mathrm{R}}(\boldsymbol{k}) p^{i j \mathrm{R}}(\boldsymbol{k}) & =p_{i j}^{\mathrm{L}}(\boldsymbol{k}) p^{i j \mathrm{~L}}(\boldsymbol{k})=0, \\
p_{i j}^{\mathrm{R}}(\boldsymbol{k}) p^{i j \mathrm{~L}}(\boldsymbol{k}) & =2, \\
k_{p} \epsilon^{m p j} p_{i j}^{A}(\boldsymbol{k}) & =-i \lambda_{\boldsymbol{k}}^{A} k p_{i}^{m}{ }_{i}^{A}(\boldsymbol{k})(\text { for } A=\mathrm{L}, \mathrm{R}),
\end{aligned}
$$

with $\lambda_{\boldsymbol{k}}^{\mathrm{R}}=+1, \lambda_{\boldsymbol{k}}^{\mathrm{L}}=-1 .{ }^{16}$ The polarization tensors satisfy $p_{i j}^{A}(\boldsymbol{k})=p_{i j}^{A}(-\boldsymbol{k})=\left(p_{i j}^{\bar{A}}(\boldsymbol{k})\right)^{*}$ $(A=\mathrm{L}, \mathrm{R} ; \overline{\mathrm{L}}=\mathrm{R}, \overline{\mathrm{R}}=\mathrm{L})$ so that the graviton satisfies the reality condition with $h_{k}^{\mathrm{R}}=$ $\left(h_{-k}^{\mathrm{L}}\right)^{*}$. We find the quadratic action of the tensor perturbation around the FRW background in the momentum space as

$$
\mathcal{S}_{\mathrm{E}-\mathrm{H}}+\mathcal{S}_{\mathrm{eff}} \simeq \frac{M_{\mathrm{Pl}}^{2}}{4} \int d \eta d^{3} k \sum_{A=L, R} a^{2}(\eta)\left[1+\lambda_{k}^{A} \frac{k}{24 \pi^{2} M_{\mathrm{Pl}}^{2}} \frac{\mu_{5}}{a(\eta)}\right]\left(\left|\left(h_{k}^{A}\right)^{\prime}\right|^{2}-k^{2}\left|h_{k}^{A}\right|^{2}\right),
$$

where the prime denotes the derivative with respect to the conformal time $\eta$. Requiring the absence of ghost-like and strong-coupling modes below the Planck scale for the physical momentum $k / a(\eta)$, we obtain the same upper bound on the value of chiral chemical potential as eq. (3.5).

We are interested in the early epoch where the primordial gravitational waves are generated by a dynamical mechanism and the Universe eventually consists of chiral asymmetric thermal plasma. We here investigate the gravitational wave propagation in the radiation-dominated Universe without any entropy production, ${ }^{17}$ in which the scale factor evolves with time as $a(\eta)=a_{0}\left(\eta / \eta_{0}\right)$ and the temperature of the Universe dilutes roughly as $T \propto a^{-1}(\eta)$. We take $\eta=\eta_{0}$ to be the "initial" time and does not take into account the process to generate the initial asymmetry. Well after the generation of gravitational waves and the source term has disappeared, the mode equation is written as

$$
\begin{aligned}
\left(h_{k}^{A}\right)^{\prime \prime}+\frac{2}{\eta}\left(h_{k}^{A}\right)^{\prime}+k^{2} h_{k}^{A} & =-\frac{\lambda_{k}^{A} k}{24 \pi^{2} M_{\mathrm{Pl}}^{2}}\left\{\frac{\mu_{5} \eta_{0}}{a_{0} \eta}\left(\frac{d^{2}}{d \eta^{2}}+\frac{2}{\eta} \frac{d}{d \eta}+k^{2}\right)+\left(\frac{\mu_{5} \eta_{0}}{a_{0} \eta}\right)^{\prime} \frac{d}{d \eta}\right\} h_{k}^{A} \\
& \equiv-\frac{\lambda_{k}^{A} k}{24 \pi^{2} M_{\mathrm{Pl}}^{2}} \hat{F}_{k}(\eta) h_{k}^{A} .
\end{aligned}
$$

Once more, we perturbatively solve the equation of motion using the Green function method. In the FRW background, the Green function is given by

$$
G(\eta, \tilde{\eta})=\Theta(\eta-\tilde{\eta}) \tilde{\eta} \frac{\sin k(\eta-\tilde{\eta})}{k \eta},
$$

which satisfies $\left(\partial_{\eta}^{2}+(2 / \eta) \partial_{\eta}+k^{2}\right) G(\eta, \tilde{\eta})=\delta(\eta-\tilde{\eta})$. From the zero-th order solution of eq. (4.4) with $\mu_{5}=\dot{\mu}_{5}=0,{ }^{18}$ which is given by

$$
h_{\boldsymbol{k}}^{A,(0)}(\eta)=h_{\mathrm{in}}^{A}(\boldsymbol{k}) \frac{\sin k \eta}{k \eta},
$$

\footnotetext{
${ }^{16}$ Note that $\lambda_{k}^{A}$ changes its sign as $\lambda_{-k}^{\mathrm{R}}=-\lambda_{k}^{\mathrm{R}}=-1, \lambda_{-\boldsymbol{k}}^{\mathrm{L}}=-\lambda_{k}^{\mathrm{L}}=+1$, with respect to the reflection of the wave vector.

${ }^{17}$ See ref. [69] for the calculation of matter-dominated era.

${ }^{18}$ Since the other solution $h_{k}(\eta) \propto \cos k \eta /(k \eta)$ is a decaying mode at the superhorizon scales, $k \eta \ll 1$, we do not consider it here.
} 
we can evaluate the first order correction as

$$
\begin{aligned}
h_{k}^{A,(1)}(\eta)-h_{k}^{A,(0)}(\eta) & =-\frac{\lambda_{k}^{A} k}{24 \pi^{2} M_{\mathrm{Pl}}^{2}} \int_{\eta_{0}}^{\infty} d \tilde{\eta} G(\eta, \tilde{\eta}) \hat{F}_{k}(\tilde{\eta}) h_{k}^{A,(0)} \\
& =-\frac{\lambda_{\boldsymbol{k}}^{A} k h_{\mathrm{in}}^{A}(\boldsymbol{k})}{24 \pi^{2} M_{\mathrm{Pl}}^{2}} \int_{\eta_{0}}^{\eta} d \tilde{\eta}\left(\frac{\mu_{5} \eta_{0}}{a_{0} \tilde{\eta}}\right)^{\prime} \frac{\sin k(\eta-\tilde{\eta})}{k \eta}\left(\cos k \tilde{\eta}-\frac{\sin k \tilde{\eta}}{k \tilde{\eta}}\right) .
\end{aligned}
$$

Compared to the Minkowski background case (eq. (3.8)), not only the intrinsic variation of the chemical potential but also the cosmological expansion affects the propagation of gravitational waves through the term $\left(\mu_{5} / a(\eta)\right)^{\prime}$. This means that the fermion background gives the non-zero correction to the gravitational waves even when the comoving chiral asymmetry is conserved and $\mu_{5}$ just decays as $\mu_{5} \propto a^{-1} \propto \eta^{-1}$ according to the cosmic expansion. Such a background curvature dependence is ascribed to the non-conformal nature of the graviton and is obviously different from the CME where one can drop the scale factor dependence by moving to the comoving frame. We would like to emphasize that we need to take into account the effect of the cosmic expansion in order to quantify the memory effect of gravitational waves, which we have discussed in the Minkowski spacetime case. With this solution, we investigate how the cosmological expansion plays a role in the interplay between the chiral gravitational waves and chemical potential through the gravitational anomaly in section 4.2 .

\subsection{The effect of the diluting chemical potential}

As we explained in section 4.1, the cosmic expansion gives a non-trivial contribution to the evolution of the gravitational waves through the chiral gravitational effect, which is not the case in the CME where all the fields involved are conformal. In order to quantify the effect of the cosmic expansion, we investigate the behavior of the chiral plasma and gravitational waves in the expanding background in the absence of the chirality-changing interactions. As is in the Minkowski spacetime case, let us consider a plasma with a chiral chemical potential $\mu_{5}(\ll T)$ or $n_{5} \simeq \mu_{5} T^{2} / 6$. Note that the temperature scales as $T \propto a^{-1}(\eta)$ in the expanding background. We divide them into the background that is simply diluted by the cosmic expansion and the modulation due to the backreaction as

$$
\mu_{5}(\eta) \simeq \frac{a_{0}}{a(\eta)}\left(\mu_{5}^{(0)}+\mu_{5}^{(1)}(\eta)\right) .
$$

Here $\mu_{5}^{(0)}$ is a constant that represents the initial chiral asymmetry of the system whereas the modulation due to the backreaction $\mu_{5}^{(1)}$ is expected to be sufficiently small. The leading correction to the gravitational waves caused by the cosmological dilution of the chemical potential is obtained by substituting $\mu_{5} \simeq \mu_{5}^{(0)}\left(a_{0} / a(\eta)\right)$ into eq. (4.7):

$$
\begin{aligned}
h_{\boldsymbol{k}}^{A,(1)}(\eta) & =h_{\mathrm{in}}^{A}(\boldsymbol{k})\left\{\frac{\sin k \eta}{k \eta}-\frac{\lambda_{k}^{A} k}{24 \pi^{2} M_{\mathrm{Pl}}^{2}} \int_{\eta_{0}}^{\eta} d \tilde{\eta}\left(\frac{a_{0} \mu_{5}^{(0)}}{a(\tilde{\eta})^{2}}\right)^{\prime} \frac{\sin k(\eta-\tilde{\eta})}{k \eta}\left(\cos k \tilde{\eta}-\frac{\sin k \tilde{\eta}}{k \tilde{\eta}}\right)\right\} \\
& =h_{\mathrm{in}}^{A}(\boldsymbol{k})\left\{\frac{\sin k \eta}{k \eta}-\frac{\lambda_{k}^{A} k \mu_{5}^{(0)}}{24 \pi^{2} M_{\mathrm{Pl}}^{2} a_{0}} \int_{\eta_{0}}^{\eta} d \tilde{\eta}\left(\frac{\eta_{0}^{2}}{\tilde{\eta}^{2}}\right)^{\prime} \frac{\sin k(\eta-\tilde{\eta})}{k \eta}\left(\cos k \tilde{\eta}-\frac{\sin k \tilde{\eta}}{k \tilde{\eta}}\right)\right\} \\
& \equiv h_{\mathrm{in}}^{A}(\boldsymbol{k})\left\{\frac{\sin k \eta}{k \eta}-\frac{\lambda_{k}^{A} k \mu_{5}^{(0)}}{24 \pi^{2} M_{\mathrm{Pl}}^{2} a_{0}} f(k, \eta)\right\},
\end{aligned}
$$


where we have defined $f(k, \eta)$ for the notational convenience. Here the first order correction $\delta h_{\mathbf{k}}^{A}(\eta) \equiv h_{\boldsymbol{k}}^{A,(1)}(\eta)-h_{\boldsymbol{k}}^{A,(0)}(\eta)=\frac{\lambda_{k}^{A} k \mu_{5}^{(0)}}{24 \pi^{2} M_{\mathrm{Pl}}^{2} a_{0}} f(k, \eta) h_{\mathrm{in}}^{A}(\boldsymbol{k})$ is the linear order in $\mu_{5}^{(0)}$. Since apparently $f(k, \eta)$ is nonzero in general and $\lambda_{k}^{A}$ has opposite signs for the left- and rightpolarization modes, we conclude that the dilution of the chiral chemical potential due to the cosmic expansion also causes the gravitational birefringence as expected.

Before investigating the properties of the correction to gravitational wave represented by $f(\eta)$, we need to confirm that the backreaction is sufficiently small, which guarantees the validity of the treatment in the above. Let us investigate the anomaly equation to quantify the backreaction to the chiral asymmetry carried by the fermions. The modified gravitational waves contribute to the evolution of the chiral chemical potential through the gravitational anomaly. With the perturbative expansion, the anomaly equation is rewritten as

$$
\partial_{\eta}\left(\mu_{5}^{(1)}\right) \simeq-\frac{6}{a_{0} a^{2} T^{2}} \frac{1}{12(4 \pi)^{2}} a^{4}\langle R \tilde{R}\rangle
$$

where we have used $a T=$ const. Note that $\langle R \tilde{R}\rangle$ does not depend on $\mu_{5}^{(1)}$ but on $\mu_{5}^{(0)}$ in our perturbative treatment. Here the Chern-Pontryagin density can be expanded as

$a^{4}\langle R \tilde{R}\rangle=\frac{2}{V} \partial_{\eta}\left[\int \frac{d^{3} k}{(2 \pi)^{3}}\left\{k\left(\left|\left(h_{\mathbf{k}}^{\mathrm{R},(1)}(\eta)\right)^{\prime}\right|^{2}-\left|\left(h_{\mathbf{k}}^{\mathrm{L},(1)}(\eta)\right)^{\prime}\right|^{2}\right)-k^{3}\left(\left|h_{\mathbf{k}}^{\mathrm{R},(1)}(\eta)\right|^{2}-\left|h_{\mathbf{k}}^{\mathrm{L},(1)}(\eta)\right|^{2}\right)\right\}\right]$,

where $V \equiv \int d^{3} x$ is the spatial volume of the system. In the case where the chirality imbalance of gravitational waves is absent in the initial distribution, namely, $\left|h_{\mathrm{in}}^{L}(\mathbf{k})\right|^{2}=$ $\left|h_{\mathrm{in}}^{R}(\mathbf{k})\right|^{2}=\left|h_{0}(\mathbf{k})\right|^{2}$, the leading order terms cancel each other and the term linear in $\mu_{5}^{(0)}$ becomes the lowest contribution as

$$
a^{4}\langle R \tilde{R}\rangle=\frac{2}{V} \partial_{\eta}\left[\int \frac{d^{3} k}{(2 \pi)^{3}} \frac{k^{3} \mu_{5}^{(0)}\left|h_{0}(\mathbf{k})\right|^{2}}{6 \pi^{2} M_{\mathrm{Pl}}^{2} a_{0}}\left\{\frac{\sin k \eta}{\eta} f(k, \eta)-\left(\frac{\cos k \eta}{k \eta}-\frac{\sin k \eta}{k^{2} \eta^{2}}\right) f^{\prime}(k, \eta)\right\}+\cdots\right] .
$$

Here the ellipses denote the higher order terms in $\delta h_{\mathbf{k}}^{A}$ and hence of $\mathcal{O}\left(\left(\mu_{5}^{(0)}\right)^{2}\right)$.

For simplicity, let us consider the case where the initial spectrum is localized in a specific scale $k_{0}$ as $\left|h_{0}(\mathbf{k})\right|^{2}=A^{2} V \delta\left(\boldsymbol{k}-\boldsymbol{k}_{0}\right)$, which corresponds to the case we discussed in the Minkowski spacetime (section 3.1). The $k$-integration in eq. (4.12) can be performed as

$$
a^{4}\langle R \tilde{R}\rangle=2 \partial_{\eta}\left[\frac{k_{0}^{3} \mu_{5}^{(0)} A^{2}}{48 \pi^{5} M_{\mathrm{Pl}}^{2} a_{0}}\left\{\frac{\sin k_{0} \eta}{\eta} f\left(k_{0}, \eta\right)-\left(\frac{\cos k_{0} \eta}{k_{0} \eta}-\frac{\sin k_{0} \eta}{k_{0}^{2} \eta^{2}}\right) f^{\prime}\left(k_{0}, \eta\right)\right\}\right]+\mathcal{O}\left(\left(\mu_{5}^{(0)}\right)^{2}\right) .
$$

Now, by comparing this with eq. (4.10), the formal expression of the first order correction to the chemical potential is given by

$$
\mu_{5}^{(1)}(\eta) \simeq-\frac{A^{2} \mu_{5}^{(0)}}{6(2 \pi)^{7}} \frac{k_{0}^{2}}{a^{2} T^{2}} \frac{k_{0}^{2}}{a_{0}^{2} M_{\mathrm{Pl}}^{2}}\left\{\frac{\sin k_{0} \eta}{k_{0} \eta} f\left(k_{0}, \eta\right)-\left(\frac{\cos k_{0} \eta}{k_{0} \eta}-\frac{\sin k_{0} \eta}{k_{0}^{2} \eta^{2}}\right) \frac{f^{\prime}\left(k_{0}, \eta\right)}{k_{0}}\right\}+C_{0}
$$

where $C_{0}$ is the integration constant to make $\mu_{5}^{(1)}\left(\eta_{0}\right)=0$. As expected, there appears a prefactor $A^{2} k_{0}^{4} / a_{0}^{2} a^{2} T^{2} M_{\mathrm{Pl}}^{2}$, which is similar to the factor $\delta$ in eq. (3.12). This is smaller than the unity if $A$ and $k_{0}$ are sufficiently small as in the Minkowski spacetime case. ${ }^{19}$ Let

\footnotetext{
${ }^{19}$ Once more, $k_{0}$ should not be too small so that the non-local effects can be neglected.
} 
us assume that $k_{0}$ lies in the superhorizon scale at $\eta_{0}, k_{0} \eta_{0} \ll 1$, and then re-enter the horizon during the radiation-dominated era. In this case, the late time behavior of $f\left(k_{0}, \eta\right)$ can be expressed as

$$
f\left(k_{0}, \eta\right) \simeq-\frac{k_{0} \eta_{0}}{9} \frac{\eta_{0}}{\eta}\left\{3 \pi \cos \left(k_{0} \eta\right)+\left(6 \gamma-5+6 \log \left(2 k_{0} \eta_{0}\right)\right) \sin \left(k_{0} \eta\right)\right\}\left(1+\mathcal{O}\left(\frac{1}{k_{0}^{2} \eta^{2}}\right)\right)
$$

where $\gamma$ is the Euler-Mascheroni constant. From these equations, we can read off the damped oscillating behavior of $\mu_{5}^{(1)}$, whose amplitude decays as $\mu_{5}^{(1)} \propto \eta^{-2} \propto a(\eta)^{-2}$. This implies that the cosmological dilution of chiral chemical potential actually causes the continual conversion between the chirality carried by fermions and the helicity of the gravitational waves as we observed in the Minkowski spacetime in section 3.2. However, the associated oscillation of the chiral chemical potential decays much faster than the dilution itself. In addition to the smallness of the prefactor $A^{2} k_{0}^{4} / a_{0}^{2} a^{2} T^{2} M_{\mathrm{Pl}}^{2}$, this rapid decay ensures that the backreaction can safely be neglected in the propagation of the gravitational waves under the diluting chiral chemical potential in the FRW spacetime. This behavior is in contrast to that in the models in the Minkowski spacetime where the backreaction eventually dominates the dynamics of chiral chemical potential.

Now we have confirmed the smallness of the backreaction, before closing let us investigate the propagation of the gravitational waves under the expanding background in more detail. From eq. (4.15), the late time behavior of the corrected gravitational wave is explicitly given by

$$
h^{L, R}(\eta) \sim \frac{A}{k_{0} \eta}\left(\sin k_{0} \eta \pm \frac{k_{0}^{3} \eta_{0}^{2} \mu_{5}^{(0)}}{216 \pi^{2} M_{\mathrm{Pl}}^{2} a_{0}^{2}}\left\{3 \pi \cos \left(k_{0} \eta\right)+\left(6 \gamma-5+6 \log \left(2 k_{0} \eta_{0}\right)\right) \sin \left(k_{0} \eta\right)\right\}\right)
$$

for the superhorizon mode at $\eta_{0}, k_{0} \eta_{0} \ll 1$, where the sign $+(-)$ is for the left (right) mode. The first term in the parentheses corresponds to the injected gravitational wave and the second term is the modulation caused by the chiral gravitational effect. Since both have the same scaling law, $\propto 1 / \eta$, the relative amplitude of the modulation does not decay (or grow). Therefore, we conclude that in principle the imprints of the cosmological dilution of the chiral plasma can be read from the gravitational wave spectrum, as expected from the discussion in section 3. If the primordial gravitational waves are produced in the early Universe and the Universe has experienced some epoch where non-zero chiral asymmetry of the fermions existed, we expect that such a gravitational birefringence is generated and remains until today. However, this in turn means that the memory of the intrinsic evolution of the chemical potential imprinted on the gravitational waves, which we discussed in section 3.1, is considerably affected by the cosmological expansion. Therefore, we need to sum up such contributions in order to give a precise prediction on the gravitational birefringence. Although the summation would result in small correction and it would not be possible to detect such an effect within the current technology, the memory effect of the chiral imbalance may enable us to capture the dynamics of primordial thermal plasma through the future gravitational wave observation. 


\section{Discussion}

In this paper, we investigate the chiral gravitational effect in the time-dependent asymmetric fermion background by solving the equation of motion for the gravitational waves with the induced gravitational Chern-Simons term and evaluating its backreaction through the anomaly equation. As we discussed in section 2, the formally covariant expression of the effective action (2.5) enables us to consider the local anomalous contribution in such a situation, for high momentum gravitational waves $k \gtrsim \mu_{5}, T$. Compared to the CME, there are two specific features in the case of the chiral gravitational effect. First, as explicitly shown for the Minkowski spacetime in section 2, the induced energy momentum tensor shows $\dot{\mu}_{5}$ dependence, which is absent in the case of CME. By solving the corrected equation of motion for the gravitational waves in section 3.1, we have found that the gravitational birefringence takes place according to the evolution of $\dot{\mu}_{5}$ during the propagation. This implies that the dynamics of the primordial chiral plasma is imprinted on the gravitational waves emitted before the generation of the chiral asymmetry through the chiral gravitational effect. We also consider the backreaction in section 3.2, where we have assumed that the time variation of $\mu_{5}$ is sourced by the external chirality-changing processes. We confirm that the memory, or the helicity accumulated in the gravitational waves, is dominated by the source contribution in the early times. We also find that the backreaction typically results in the oscillatory conversion between the chirality carried by fermions and the helicity of the gravitational waves. This oscillatory behavior, driven by the external chirality-changing process, is in contrast with the CME where the CPI is triggered by the constant $\mu_{5}$. The second feature is that cosmic expansion gives a non-trivial contribution due to the non-conformal nature of the graviton. As we demonstrate in section 4.1 and 4.2, a diluting chemical potential also causes the gravitational birefringence and drives oscillatory conversion between the chirality and the helicity as a backreaction. While the correction of the gravitational waves has the same scaling law as the free gravitational waves, $\propto 1 / \eta$, the backreaction to the chiral plasma turns out to decay rapidly as $\propto 1 / \eta^{2}$ and is highly suppressed by a small overall factor as in the Minkowski spacetime case. We would like to stress that there is no violent amplification of the gravitational field like CPI as long as the local description of the chiral gravitational effect is concerned, which ensures the backreaction to be negligible in our treatment. This difference originates from the different structure of the Chern-Simons term, or the symmetry of the system. While the Chern-Simons term causes the tachyonic instability for the spin-1 gauge field within the region of validity of the effective field theory, gravitational Chern-Simons term leads to the ghost instability for very high momentum modes. Since the effective field theory description breaks down in such a regime, we restrict our study in the scale much below the Planck or the cut-off scale where the Chern-Simons term correction can be perturbatively included, and reasonably avoid the appearance of ghost modes.

Let us make a qualitative discussion on the chiral gravitational effect in cosmology. If one considers some scenarios of baryogenesis like the GUT baryogenesis, for example, $\mu_{5}$ can be generated at the time of the baryogenesis and eventually decays when the Yukawa interaction gets in equilibrium $[29,33,47]$. In this case, non-trivial time dependence on 
$\mu_{5}$ is given by the generation, decay, and the cosmological dilution. The memory of the chiral imbalance imprinted on the gravitational waves is determined by the combination of those contributions. As we demonstrated in section 3.2 and section 4.2, the backreaction can be neglected for the propagation of the gravitational waves. It would enable us to investigate the memory effect of the gravitational waves for various models of the baryogenesis or leptogenesis without complicated discussions. Such imprints of the primordial chirality violation in the plasma might be observed by the recently proposed high frequency gravitational wave detectors [85-88] since the Chern-Simons term-like local contribution we investigated here becomes dominant for the high momentum region $k \gtrsim T$.

We would like to add a comment on the possibility that this memory effect in gravitational wave might become the probe of the dark sector. Since gravity couples to the any kinds of matter fields, the chiral gravitational effect can take place due to the chiral flipping process even in the dark sector. Although such effects would be too small to be detected with present gravitational detectors, it is worth investigating such chiral gravitational waves in detail. This possibility motivates us to make a further study of the chiral gravitational effects including non-local effects in general backgrounds, which we do not address in this work.

Finally, we would like to make a comment on the limitation of our study and the possible extension of it. First, we considered monotonic plane gravitational waves as the incoming wave. In more realistic case, especially for the application to cosmology, we need to take into account the stochasticity of the gravitational waves and the effect of their spectrum. This would become important when we also take into account the spatial dependence of the chiral chemical potential, which is neglected in this study. Moreover, as we mentioned in section 2, induced gravitational Chern-Simons term cannot reproduce the non-local contributions to the anomalous dispersion, which includes, for example, the thermal correction derived in the study of static thermal background [64, 65]. Although the Chern-Simons term and the spurious field enable us to appropriately take into account the time dependence of chemical potential of the background, the anomalous dispersion of the gravitational waves arising from this term becomes leading only when the conditions $\mu_{5} \lesssim T \lesssim k$ are satisfied. If one considers the low momentum region $k \lesssim T$ instead, where the ground-based and space-born gravitational detector become sensitive, thermal corrections would dominate the anomalous dispersion relation of the gravitational waves. In this sense, extensions of our study to include thermal non-local corrections properly is necessary to probe the chiral thermal plasma in the early Universe by the present and planned gravitational wave detectors. However, incorporating thermal correction into our formally covariant effective Lagrangian is not so straightforward since the thermal effect associated to the global nature of background appears as the non-local contribution, as we mentioned in section 2. To achieve such an extension, we need to take different approaches beyond the covariant local Lagrangian formalism we have taken. We leave such extensions for future study. 


\section{Acknowledgments}

The authors thank Naoki Yamamoto for useful discussions. This work was partially supported by JSPS KAKENHI Grant-in-Aid for Scientific Research (C) JP19K03842 (KK) and Innovative Area 19H04610(KK). JK is supported by JSPS KAKENHI, Grant-in-Aid for JSPS Fellows 20J21866 and research program of the Leading Graduate Course for Frontiers of Mathematical Sciences and Physics (FMSP). YY is supported by JSPS KAKENHI, Grant-in-Aid for JSPS Fellows JP19J00494.

Open Access. This article is distributed under the terms of the Creative Commons Attribution License (CC-BY 4.0), which permits any use, distribution and reproduction in any medium, provided the original author(s) and source are credited.

\section{References}

[1] A. Vilenkin, Equilibrium parity violating current in a magnetic field, Phys. Rev. D 22 (1980) 3080 [INSPIRE].

[2] H.B. Nielsen and M. Ninomiya, Adler-Bell-Jackiw anomaly and Weyl fermions in crystal, Phys. Lett. B 130 (1983) 389 [InSPIRE].

[3] A.Y. Alekseev, V.V. Cheianov and J. Fröhlich, Universality of transport properties in equilibrium, Goldstone theorem and chiral anomaly, Phys. Rev. Lett. 81 (1998) 3503 [cond-mat/9803346] [INSPIRE].

[4] D.T. Son and A.R. Zhitnitsky, Quantum anomalies in dense matter, Phys. Rev. D 70 (2004) 074018 [hep-ph/0405216] [INSPIRE].

[5] K. Fukushima, D.E. Kharzeev and H.J. Warringa, The Chiral Magnetic Effect, Phys. Rev. D 78 (2008) 074033 [arXiv: 0808.3382] [INSPIRE].

[6] A.A. Zyuzin and A.A. Burkov, Topological response in Weyl semimetals and the chiral anomaly, Phys. Rev. B 86 (2012) 115133 [arXiv:1206.1868] [INSPIRE].

[7] P. Goswami and S. Tewari, Axionic field theory of $(3+1)$-dimensional Weyl semimetals, Phys. Rev. B $8 \mathbf{8}$ (2013) 245107 [arXiv:1210.6352] [InSPIRE].

[8] Y. Chen, S. Wu and A.A. Burkov, Axion response in Weyl semimetals, Phys. Rev. B 88 (2013) 125105 [arXiv: 1306. 5344] [INSPIRE].

[9] G. Basar, D.E. Kharzeev and H.-U. Yee, Triangle anomaly in Weyl semimetals, Phys. Rev. B 89 (2014) 035142 [arXiv: 1305.6338] [INSPIRE].

[10] P. Hosur and X. Qi, Recent developments in transport phenomena in Weyl semimetals, Comptes Rendus Physique 14 (2013) 857 [arXiv:1309.4464] [INSPIRE].

[11] K. Landsteiner, Anomalous transport of Weyl fermions in Weyl semimetals, Phys. Rev. B 89 (2014) 075124 [arXiv: 1306 .4932] [INSPIRE].

[12] M.N. Chernodub, A. Cortijo, A.G. Grushin, K. Landsteiner and M.A.H. Vozmediano, Condensed matter realization of the axial magnetic effect, Phys. Rev. B 89 (2014) 081407 [arXiv: 1311.0878] [INSPIRE].

[13] D.E. Kharzeev and H.-U. Yee, Chiral Magnetic Wave, Phys. Rev. D 83 (2011) 085007 [arXiv: 1012.6026] [INSPIRE]. 
[14] Y. Burnier, D.E. Kharzeev, J. Liao and H.-U. Yee, Chiral magnetic wave at finite baryon density and the electric quadrupole moment of quark-gluon plasma in heavy ion collisions, Phys. Rev. Lett. 107 (2011) 052303 [arXiv:1103.1307] [InSPIRE].

[15] M. Hongo, Y. Hirono and T. Hirano, Anomalous-hydrodynamic analysis of charge-dependent elliptic flow in heavy-ion collisions, Phys. Lett. B 775 (2017) 266 [arXiv:1309.2823] [INSPIRE].

[16] H.-U. Yee and Y. Yin, Realistic Implementation of Chiral Magnetic Wave in Heavy Ion Collisions, Phys. Rev. C 89 (2014) 044909 [arXiv:1311.2574] [InSPIRE].

[17] Y. Hirono, T. Hirano and D.E. Kharzeev, The chiral magnetic effect in heavy-ion collisions from event-by-event anomalous hydrodynamics, arXiv:1412.0311 [INSPIRE].

[18] X.-G. Huang, Electromagnetic fields and anomalous transports in heavy-ion collisions - A pedagogical review, Rept. Prog. Phys. 79 (2016) 076302 [arXiv:1509.04073] [InSPIRE].

[19] D.E. Kharzeev, J. Liao, S.A. Voloshin and G. Wang, Chiral magnetic and vortical effects in high-energy nuclear collisions - A status report, Prog. Part. Nucl. Phys. 88 (2016) 1 [arXiv: 1511.04050] [INSPIRE].

[20] S. Shi, Y. Jiang, E. Lilleskov and J. Liao, Anomalous Chiral Transport in Heavy Ion Collisions from Anomalous-Viscous Fluid Dynamics, Annals Phys. 394 (2018) 50 [arXiv: 1711.02496] [INSPIRE].

[21] J. Charbonneau and A. Zhitnitsky, Topological Currents in Neutron Stars: Kicks, Precession, Toroidal Fields, and Magnetic Helicity, JCAP 08 (2010) 010 [arXiv: 0903.4450] [INSPIRE].

[22] A. Ohnishi and N. Yamamoto, Magnetars and the Chiral Plasma Instabilities, arXiv: 1402.4760 [INSPIRE].

[23] D. Grabowska, D.B. Kaplan and S. Reddy, Role of the electron mass in damping chiral plasma instability in Supernovae and neutron stars, Phys. Rev. D 91 (2015) 085035 [arXiv: 1409.3602] [INSPIRE].

[24] M. Kaminski, C.F. Uhlemann, M. Bleicher and J. Schaffner-Bielich, Anomalous hydrodynamics kicks neutron stars, Phys. Lett. B $\mathbf{7 6 0}$ (2016) 170 [arXiv:1410.3833] [INSPIRE].

[25] G. Sigl and N. Leite, Chiral Magnetic Effect in Protoneutron Stars and Magnetic Field Spectral Evolution, JCAP 01 (2016) 025 [arXiv: 1507.04983] [INSPIRE].

[26] N. Yamamoto, Chiral transport of neutrinos in supernovae: Neutrino-induced fluid helicity and helical plasma instability, Phys. Rev. D 93 (2016) 065017 [arXiv:1511.00933] [InSPIRE].

[27] Y. Masada, K. Kotake, T. Takiwaki and N. Yamamoto, Chiral magnetohydrodynamic turbulence in core-collapse supernovae, Phys. Rev. D 98 (2018) 083018 [arXiv:1805.10419] [INSPIRE].

[28] D. Grasso and H.R. Rubinstein, Magnetic fields in the early universe, Phys. Rept. 348 (2001) 163 [astro-ph/0009061] [INSPIRE].

[29] B.A. Campbell, S. Davidson, J.R. Ellis and K.A. Olive, On the baryon, lepton flavor and right-handed electron asymmetries of the universe, Phys. Lett. B 297 (1992) 118 [hep-ph/9302221] [INSPIRE].

[30] M. Joyce and M.E. Shaposhnikov, Primordial magnetic fields, right-handed electrons, and the Abelian anomaly, Phys. Rev. Lett. 79 (1997) 1193 [astro-ph/9703005] [INSPIRE]. 
[31] Y. Akamatsu and N. Yamamoto, Chiral Plasma Instabilities, Phys. Rev. Lett. 111 (2013) 052002 [arXiv: 1302 . 2125] [INSPIRE].

[32] H. Tashiro, T. Vachaspati and A. Vilenkin, Chiral Effects and Cosmic Magnetic Fields, Phys. Rev. D 86 (2012) 105033 [arXiv:1206.5549] [INSPIRE].

[33] K. Kamada, Return of grand unified theory baryogenesis: Source of helical hypermagnetic fields for the baryon asymmetry of the universe, Phys. Rev. D 97 (2018) 103506 [arXiv: 1802.03055] [INSPIRE].

[34] M. Giovannini and M.E. Shaposhnikov, Primordial magnetic fields, anomalous isocurvature fluctuations and big bang nucleosynthesis, Phys. Rev. Lett. 80 (1998) 22 [hep-ph/9708303] [INSPIRE].

[35] M. Giovannini and M.E. Shaposhnikov, Primordial hypermagnetic fields and triangle anomaly, Phys. Rev. D 57 (1998) 2186 [hep-ph/9710234] [INSPIRE].

[36] K. Bamba, Baryon asymmetry from hypermagnetic helicity in dilaton hypercharge electromagnetism, Phys. Rev. D 74 (2006) 123504 [hep-ph/0611152] [INSPIRE].

[37] T. Fujita and K. Kamada, Large-scale magnetic fields can explain the baryon asymmetry of the Universe, Phys. Rev. D 93 (2016) 083520 [arXiv:1602.02109] [InSPIRE].

[38] K. Kamada and A.J. Long, Baryogenesis from decaying magnetic helicity, Phys. Rev. D 94 (2016) 063501 [arXiv:1606.08891] [INSPIRE].

[39] K. Kamada and A.J. Long, Evolution of the Baryon Asymmetry through the Electroweak Crossover in the Presence of a Helical Magnetic Field, Phys. Rev. D 94 (2016) 123509 [arXiv: 1610.03074] [INSPIRE].

[40] J. Schober et al., Laminar and turbulent dynamos in chiral magnetohydrodynamics. II. Simulations, Astrophys. J. 858 (2018) 124 [arXiv:1711.09733] [INSPIRE].

[41] D.G. Figueroa, A. Florio and M. Shaposhnikov, Chiral charge dynamics in Abelian gauge theories at finite temperature, JHEP 10 (2019) 142 [arXiv:1904.11892] [INSPIRE].

[42] M. Mace, N. Mueller, S. Schlichting and S. Sharma, Chiral Instabilities and the Onset of Chiral Turbulence in QED Plasmas, Phys. Rev. Lett. 124 (2020) 191604 [arXiv:1910.01654] [INSPIRE].

[43] S. Anand, J.R. Bhatt and A.K. Pandey, Chiral plasma instability and primordial gravitational waves, Eur. Phys. J. C 79 (2019) 119 [arXiv: 1801.00650] [INSPIRE].

[44] A. Brandenburg, Y. He, T. Kahniashvili, M. Rheinhardt and J. Schober, Relic gravitational waves from the chiral magnetic effect, Astrophys. J. 911 (2021) 110 [arXiv:2101.08178] [INSPIRE].

[45] V. Domcke and K. Mukaida, Gauge Field and Fermion Production during Axion Inflation, JCAP 11 (2018) 020 [arXiv: 1806.08769] [InSPIRE].

[46] V. Domcke, B. von Harling, E. Morgante and K. Mukaida, Baryogenesis from axion inflation, JCAP 10 (2019) 032 [arXiv:1905.13318] [INSPIRE].

[47] V. Domcke, K. Kamada, K. Mukaida, K. Schmitz and M. Yamada, Wash-In Leptogenesis, arXiv:2011.09347 [INSPIRE].

[48] A. Sadofyev and S. Sen, Chiral Anomalous Dispersion, JHEP 02 (2018) 099 [arXiv: 1712.03956] [INSPIRE].

[49] A. Avdoshkin and R. Sharipov, Chiral transport in curved spacetime via holography, arXiv: 2009.13796 [INSPIRE]. 
[50] L. Álvarez-Gaumé and E. Witten, Gravitational Anomalies, Nucl. Phys. B 234 (1984) 269 [INSPIRE].

[51] K. Landsteiner, E. Megias and F. Pena-Benitez, Gravitational Anomaly and Transport, Phys. Rev. Lett. 107 (2011) 021601 [arXiv:1103.5006] [InSPIRE].

[52] S.H.-S. Alexander, M.E. Peskin and M.M. Sheikh-Jabbari, Leptogenesis from gravity waves in models of inflation, Phys. Rev. Lett. 96 (2006) 081301 [hep-th/0403069] [InSPIRE].

[53] D.H. Lyth, C. Quimbay and Y. Rodriguez, Leptogenesis and tensor polarisation from a gravitational Chern-Simons term, JHEP 03 (2005) 016 [hep-th/0501153] [INSPIRE].

[54] W. Fischler and S. Paban, Leptogenesis from Pseudo-Scalar Driven Inflation, JHEP 10 (2007) 066 [arXiv:0708.3828] [inSPIRE].

[55] K. Kamada, J. Kume, Y. Yamada and J. Yokoyama, Gravitational leptogenesis with kination and gravitational reheating, JCAP 01 (2020) 016 [arXiv: 1911.02657] [INSPIRE].

[56] S. Kawai and J. Kim, Gauss-Bonnet Chern-Simons gravitational wave leptogenesis, Phys. Lett. B 789 (2019) 145 [arXiv:1702.07689] [INSPIRE].

[57] K. Kamada, J. Kume and Y. Yamada, Renormalization in gravitational leptogenesis with pseudo-scalar-tensor coupling, JCAP 10 (2020) 030 [arXiv: 2007.08029] [INSPIRE].

[58] A. Maleknejad, M. Noorbala and M.M. Sheikh-Jabbari, Leptogenesis in inflationary models with non-Abelian gauge fields, Gen. Rel. Grav. 50 (2018) 110 [arXiv: 1208. 2807] [INSPIRE].

[59] A. Maleknejad, Chiral Gravity Waves and Leptogenesis in Inflationary Models with non-Abelian Gauge Fields, Phys. Rev. D 90 (2014) 023542 [arXiv:1401.7628] [INSPIRE].

[60] R.R. Caldwell and C. Devulder, Axion Gauge Field Inflation and Gravitational Leptogenesis: A Lower Bound on B Modes from the Matter-Antimatter Asymmetry of the Universe, Phys. Rev. D 97 (2018) 023532 [arXiv: 1706.03765] [InSPIRE].

[61] A. Papageorgiou and M. Peloso, Gravitational leptogenesis in Natural Inflation, JCAP 12 (2017) 007 [arXiv: 1708.08007] [INSPIRE].

[62] P. Adshead, J.T. Giblin and Z.J. Weiner, Gravitational waves from gauge preheating, Phys. Rev. D 98 (2018) 043525 [arXiv: 1805.04550] [INSPIRE].

[63] P. Adshead, A.J. Long and E.I. Sfakianakis, Gravitational Leptogenesis, Reheating, and Models of Neutrino Mass, Phys. Rev. D 97 (2018) 043511 [arXiv:1711.04800] [INSPIRE].

[64] J.L. Manes and M. Valle, Parity violating gravitational response and anomalous constitutive relations, JHEP 01 (2013) 008 [arXiv:1211.0876] [InSPIRE].

[65] K. Jensen, R. Loganayagam and A. Yarom, Thermodynamics, gravitational anomalies and cones, JHEP 02 (2013) 088 [arXiv: 1207.5824] [INSPIRE].

[66] A.N. Redlich and L.C.R. Wijewardhana, Induced Chern-Simons Terms at High Temperatures and Finite Densities, Phys. Rev. Lett. 54 (1985) 970 [inSPIRE].

[67] D.E. Kharzeev, Chern-Simons current and local parity violation in hot QCD matter, Nucl. Phys. A 830 (2009) 543C [arXiv: 0908.0314] [InSPIRE].

[68] A. Boyarsky, J. Fröhlich and O. Ruchayskiy, Magnetohydrodynamics of Chiral Relativistic Fluids, Phys. Rev. D 92 (2015) 043004 [arXiv: 1504.04854] [inSPIRE].

[69] N.D. Barrie and A. Kobakhidze, Gravitational Instabilities of the Cosmic Neutrino Background with Non-zero Lepton Number, Phys. Lett. B 772 (2017) 459 [arXiv: 1701.00603] [INSPIRE]. 
[70] L.P. Grishchuk, Amplification of gravitational waves in an istropic universe, Zh. Eksp. Teor. Fiz. 67 (1974) 825 [INSPIRE].

[71] A.A. Starobinsky, Spectrum of relict gravitational radiation and the early state of the universe, JETP Lett. 30 (1979) 682 [INSPIRE].

[72] S.Y. Khlebnikov and I.I. Tkachev, Relic gravitational waves produced after preheating, Phys. Rev. D 56 (1997) 653 [hep-ph/9701423] [INSPIRE].

[73] R. Easther and E.A. Lim, Stochastic gravitational wave production after inflation, JCAP 04 (2006) 010 [astro-ph/0601617] [INSPIRE].

[74] R. Easther, J.T. Giblin Jr. and E.A. Lim, Gravitational Wave Production At The End Of Inflation, Phys. Rev. Lett. 99 (2007) 221301 [astro-ph/0612294] [INSPIRE].

[75] J.F. Dufaux, A. Bergman, G.N. Felder, L. Kofman and J.-P. Uzan, Theory and Numerics of Gravitational Waves from Preheating after Inflation, Phys. Rev. D 76 (2007) 123517 [arXiv: 0707.0875] [INSPIRE].

[76] A. Kosowsky, M.S. Turner and R. Watkins, Gravitational radiation from colliding vacuum bubbles, Phys. Rev. D 45 (1992) 4514 [INSPIRE].

[77] A. Kosowsky, M.S. Turner and R. Watkins, Gravitational waves from first order cosmological phase transitions, Phys. Rev. Lett. 69 (1992) 2026 [INSPIRE].

[78] A. Nicolis, Relic gravitational waves from colliding bubbles and cosmic turbulence, Class. Quant. Grav. 21 (2004) L27 [gr-qc/0303084] [INSPIRE].

[79] C. Grojean and G. Servant, Gravitational Waves from Phase Transitions at the Electroweak Scale and Beyond, Phys. Rev. D 75 (2007) 043507 [hep-ph/0607107] [InSPIRE].

[80] D. Kharzeev, Y. Kikuchi and R. Meyer, Chiral magnetic effect without chirality source in asymmetric Weyl semimetals, Eur. Phys. J. B 91 (2018) 83 [arXiv:1610.08986] [InSPIRE].

[81] S. Alexander and N. Yunes, Chern-Simons Modified General Relativity, Phys. Rept. 480 (2009) 1 [arXiv:0907.2562] [INSPIRE].

[82] J.F. Assunção, T. Mariz, J.R. Nascimento and A.Y. Petrov, Induced Chern-Simons modified gravity at finite temperature, JHEP 08 (2018) 072 [arXiv: 1805.11049] [INSPIRE].

[83] G.M. Monteiro, A.G. Abanov and V.P. Nair, Hydrodynamics with gauge anomaly: Variational principle and Hamiltonian formulation, Phys. Rev. D 91 (2015) 125033 [arXiv: 1410.4833] [INSPIRE].

[84] E. Mottola and A.V. Sadofyev, Chiral Waves on the Fermi-Dirac Sea: Quantum Superfluidity and the Axial Anomaly, Nucl. Phys. B 966 (2021) 115385 [arXiv: 1909.01974] [InSPIRE].

[85] A.M. Cruise, The potential for very high-frequency gravitational wave detection, Class. Quant. Grav. 29 (2012) 095003 [INSPIRE].

[86] A. Ito, T. Ikeda, K. Miuchi and J. Soda, Probing GHz gravitational waves with graviton-magnon resonance, Eur. Phys. J. C 80 (2020) 179 [arXiv: 1903.04843] [InSPIRE].

[87] V. Domcke and C. Garcia-Cely, Potential of radio telescopes as high-frequency gravitational wave detectors, Phys. Rev. Lett. 126 (2021) 021104 [arXiv: 2006.01161] [INSPIRE].

[88] N. Aggarwal et al., Challenges and Opportunities of Gravitational Wave Searches at MHz to GHz Frequencies, arXiv:2011.12414 [INSPIRE]. 